\title{
Expansion of Wave Rays and Fronts in Media with Inhomogeneous Structure
}

\author{
Anatoli Chigarev ${ }^{1, *}$, Ju. Chigarev² \\ ${ }^{1}$ Department Theoretical Mechanics and Mechatronics, Belarusian National Technical University, Belarus \\ ${ }^{2}$ West Pomeranian University of Technology, Szczecin, Poland
}

Copyright $(\mathrm{C} 2018$ by authors, all rights reserved. Authors agree that this article remains permanently open access under the terms of the Creative Commons Attribution License 4.0 International License

\begin{abstract}
In the article on the principles of Fermet, Huygens obtaine the differential equations in the form of Hamilton, which describe the ray trajectories and wave fronts in inhomogeneous media. Established that the vector of Poynting-Umov's determining the direction of energy propagation in inhomogeneous medium is coincident with the vector tangent to the ray. In the second part of the article established that the equations of the theory of rays' propagation in inhomogeneous media have the form of equations of nonlinear dynamics and describe the emergence of deterministic chaos in the geometry of rays for a wide variety of types of heterogeneous structures. In this case, the rays behave randomly and their description you must go to the description based on the theory of random functions and fields. In the third part of the paper is considered a model which is equivalent to the random medium and the calculation of the coordinates of the ray (the mathematical expectation and correlation functions). Understanding of these characteristics gives information about the behavior of the trajectories of the rays for these models of media. The description of the behavior of rays on the basis of the equations of statistical mechanics is discussed in the article for functions of Markov's type.
\end{abstract}

Keywords Ray, Inhomogeneous Media, Deterministic Chaos, Correlation Function, Probabilistic, Energy, Stochastic

\section{Introduction}

The purpose of this paper is to present generalizations of the results obtained for the first time in the work of the authors for moveouts of rays and wave fronts. In media where this type of inhomogeneity has place the rays be have randomly and therefore, it is necessary to switch to the probabilistic models and corresponding methods.

In the first of the article are formulated the basic equations which are obtained on the basis of the principles of Fermat, Huygens, and installed that lines of energy (vector of Poynting-Umov) and the rays path coincide. On the basis of the optical-mechanical analogy formulated the basic equations of the theory of wave propagation in the form of Hamilton are used in 2, 3 parts of the human body.

Nonlinear differential equations for ray trajectories describe deterministic chaos in the geometry of rays for wide type of an inhomogeneity. It requires a shift to a probabilistic description of the kinematics of the rays. The third part describes the model of random media described on the basis of moments (mathematical expectation and correlation functions) as well as case governmental functions of Markov type.

Simulation of wave propagation processes in inhomogeneous media is based on kinematic [1-4] and dynamic principles [5-10] for the process of ray propagation and wave surfaces (fronts) in different media.

Huygens' principle of constructing wave fronts in according to algorithm of a contact transformation is easily implemented if the perturbation region is non-concave Fig. 1.1a.

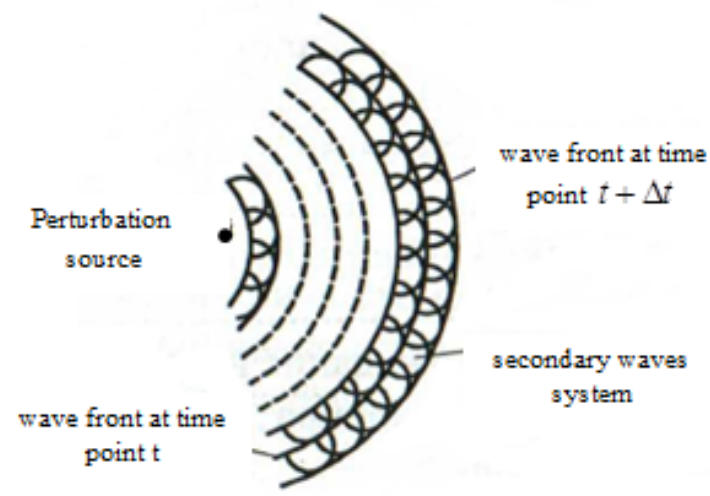

Figure 1.1a. Huygens' model for propagation of wave fronts

If the emitting area has a concavity, the construction of the wave surface is shown in Fig. 1.1b.

Presentation of the wave front in the form of the surface 
is a mathematical idealization, because in reality, a wave is a volume configuration change of the medium points during the passage of a perturbation from some initial configuration to the final. In a homogeneous isotropic medium all front points have the same speed directed along the normal $\bar{n}$, then during the time $\Delta t$ surface points are shifted by the same distance $s$ along the normal with the speed $\bar{V}$. The wave surface at time point $t+\Delta t$, constructed according to Huygens' principle as the concavity of secondary waves coincides with the surface, passing through the points lying on one and the same distance along the normal from the wave surface at time point $t$. These lines, which are orthogonal to the original radiating surface (in particular, points) in a homogeneous isotropic medium, are the rays along which the radiation energy propagates.

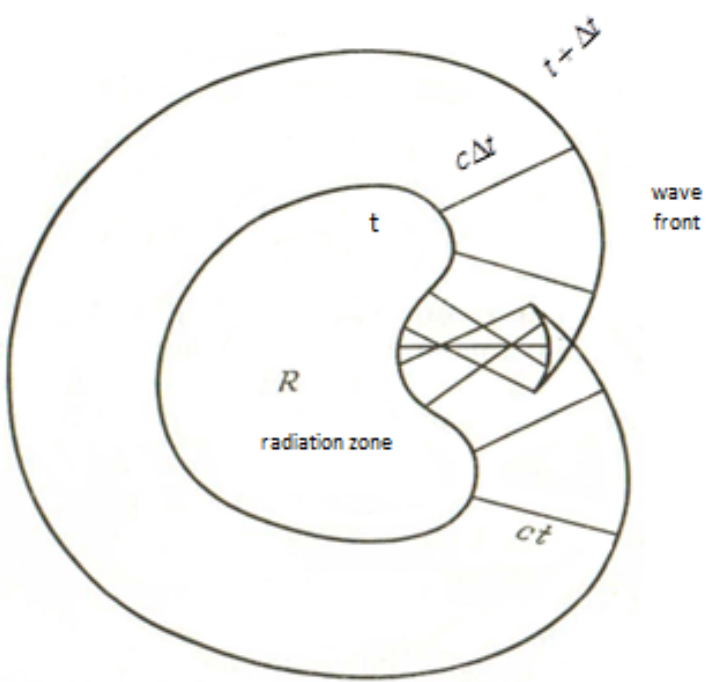

Figure 1.1b. Huygens model for propagation of wave front in case when the emining area has a concavity

According to Newton's corpuscular theory, the energy is transferred along the rays, the construction of which in homogeneous isotropic media is carried out with purely geometrical methods. Approaches of Huygens and Newton are known as the optic and mechanical analogs in analytical mechanics [1-2]. With the approach of Newton is associated the analogy of particle motion by inertia under the influence of the initial pulse and in the absence of any effects during the movement. With the approach of Huygens is associated the analogy of contact transformations in the Hamiltonian mechanics, representing a canonical transformation of generalized coordinates and momenta.

An approach based on the construction of rays is effective in solving problems of the wave kinematics by geometrical methods for homogeneous isotropic media, including the case of transmission and reflection of waves at the interface of two media, also through the lens, etc.

\section{Expansion of Rays and Fronts in Determinate Inhomogeneous Media}

In the case of inhomogeneous, anisotropic, nonlinear media Huygens' approach is more difficult to implement and Newton's approach allows solving the problem of wave propagation more effectively, if we use the kinematic principle of Farm, according to which the perturbation of the medium state at the source $M_{0}\left(x_{0}, y_{0}, z_{0}\right)$ extends to any receiver point $M\left(x_{1}, x_{2}, x_{3}\right)$ for the minimum time $\tau\left(M_{0}, M\right)$, which is the Farm's functional [1-4]

$$
\tau\left(M_{0}, M\right)=\int_{M_{0}}^{M} \frac{d \ell}{V\left(x_{1}, x_{2}, x_{3}\right)}
$$

where $V\left(x_{1}, x_{2}, x_{3}\right)$ perturbation propagation speed including $\left(x_{1}, x_{2}, x_{3}\right)$ that of inhomogeneous media, $\ell-$ the distance along the ray.

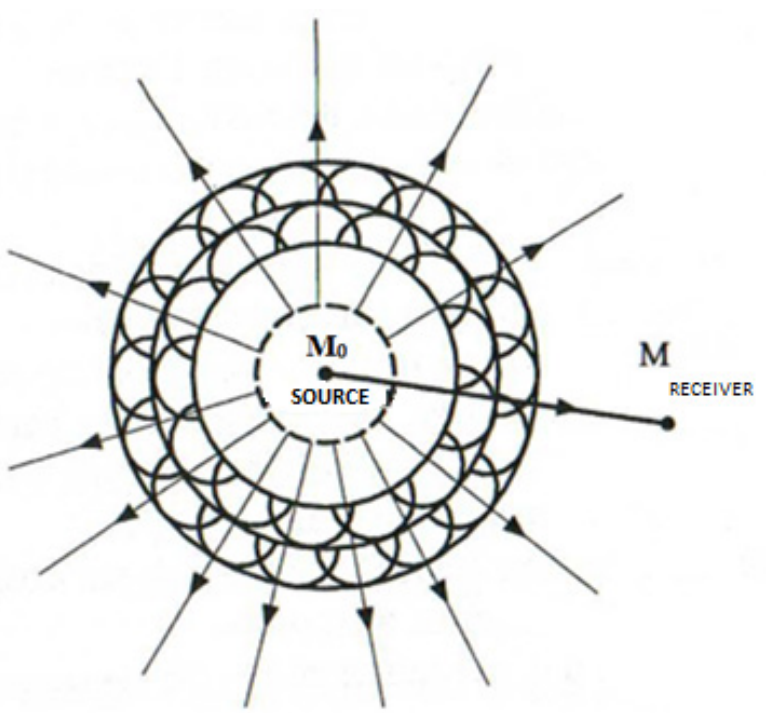

Figure 1.2. Construction scheme of rays in the Farm's model

For equation (1.1) are formulated direct and inverse tasks.

In the direct task $V\left(x_{1}, x_{2}, x_{3}\right)$ is set and is possible to build surface-isochrones $\tau\left(M_{0}, M\right)=C \quad(C$ arbitrary constant), representing a family of wave fronts.

In the inverse task of the known $\tau\left(M_{0}, M\right)$ is necessary to determine $V\left(x_{1}, x_{2}, x_{3}\right)$, namely to identify the physical and mechanical characteristics of the media (media profile).

Ray tube in a inhomogeneous medium is a figure formed by adjacent rays Fig.1.3. 


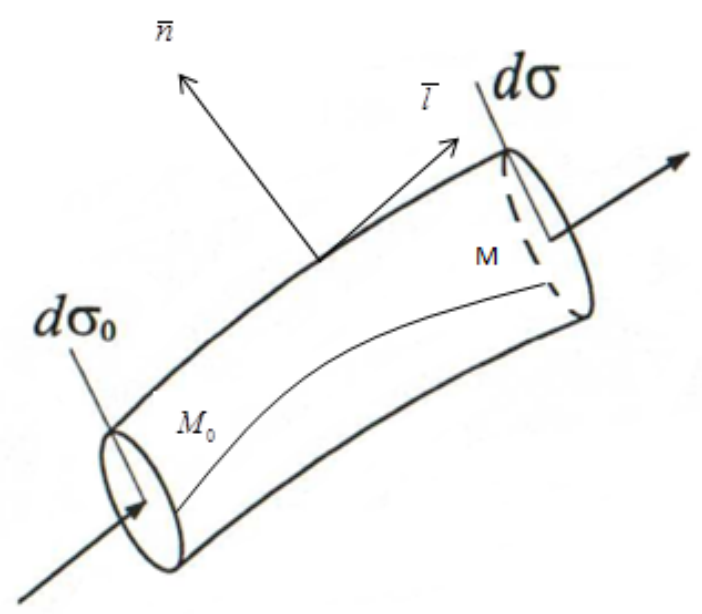

Figure 1.3. Ray tube

We denote by A function that characterizes the change in the unit ray tube cross-sectional area of the value of $d \sigma_{0}$ at the initial front to the current cross section

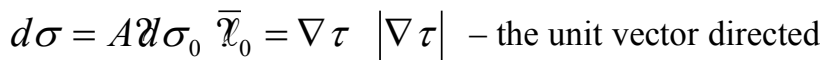
along the ray Fig. 1.4.

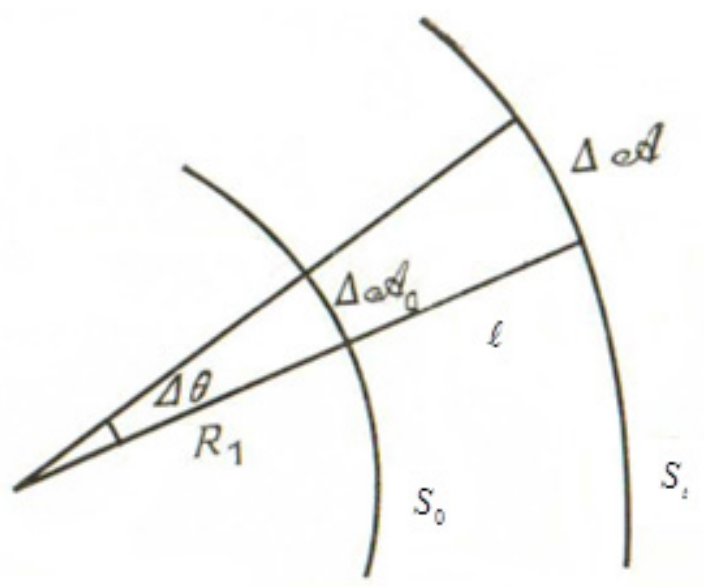

Figure 1.4. The change $\sigma$ in the ray

We calculate the integral by volume of ray tube $\Sigma_{t}$ passing to the surface integral by the formula

$$
\int_{\Sigma_{t}} d i v\left(\frac{\bar{\ell}}{A}\right) d v=\oint \frac{\bar{\ell}_{0} * \bar{n}}{A} d \ell
$$

Since on the side surface $\bar{\ell} \perp \bar{n}$, то $d \sigma A-d \sigma_{0} A_{0}=0$, hence, we obtain

$$
\operatorname{div}[(v \nabla \tau) / A]=0, \nabla \tau=\operatorname{grad} \tau
$$

Considering the ratio of

$$
v^{2}=|\nabla \tau|^{-2}
$$

we obtain the equations for finding the position and shape of the wavefront. Equation (1.4) is called the eikonal equation, and the equation (1.3) determines the change in cross section of the tube.

In particular, for the case of 2D rays and consistent positions of the front form an orthogonal curvilinear grid $\alpha, \beta$ Fig. 1.5.



Figure 1.5. The case of $2 \mathrm{D}$ rays

for which two kinematic equations are obtained

$$
\frac{1}{V} \frac{\partial A}{\partial \alpha}=\frac{\partial \theta}{\partial \beta}, \frac{1}{A} \frac{\partial V}{\partial \beta}=-\frac{\partial \theta}{\partial \alpha}
$$

where $\theta(\alpha, \beta)-$ an angle which for linear homogeneous medium does not depend on $\alpha$, because $V=V_{0}=$ const. As $A$ depends linearly on $\alpha$, the rays will be straight, and the fronts will be circles in the plane considered.

The law of energy conservation in the integral form is

$$
\frac{\partial}{\partial t} \int_{\Sigma_{t}} E d v=-\oint P_{j} n_{j} d s
$$

\section{Here $P_{j}$ - vector components of the energy density}

$\overline{\boldsymbol{P}}$ of Poynting-Umov, $E$ - the total energy density.

In the differential form of (1.6) we obtain

$$
\frac{\partial E}{\partial t}+\operatorname{div} \bar{P}=0
$$

The energy flow is directed along the speed $\bar{V}$, and hence along the rays. For an arbitrary ray tube of (1.7) follows the conservation equation

$$
\frac{\partial(E A)}{\partial t}+\frac{\partial(\bar{P} * \bar{A})}{\partial e}=0
$$

where $\ell$ - the distance along the ray. 
The eikonal equation (1.4) is a non-linear differential equation in partial derivatives of the first order type Hamilton-Jacobi, which is generally written as [11-14]

$$
H\left(\frac{\partial \tau}{\partial q_{1}}, \ldots \frac{\partial \tau}{\partial q_{n}} ; q_{1} \ldots q_{n}\right)=0
$$

where $\tau=\tau\left(q_{1} \ldots q_{n}\right)-$ the desired function, $q_{i}$ the generalized coordinates $(i=\overline{1, n})$.

Designating $\frac{\partial \tau}{\partial q_{i}}=p_{i}$ as generalized impulses, the equation (1.9) can be written as

$$
H\left(p_{i}, q_{i}\right)=0
$$

Equation characteristics (1.9) satisfy the system of ordinary differential equations

$$
\frac{d q_{i}}{\partial H / \partial p_{i}}=-\frac{d p_{i}}{\partial H / \partial q_{i}}=\frac{d \tau}{\sum_{i=1}^{n} p_{j}\left(\partial H / \partial p_{i}\right)}
$$

which can be written as

$$
\frac{d q_{i}}{d t}=\frac{\partial H}{\partial p_{i}}, \frac{d p_{i}}{d t}=-\frac{\partial H}{\partial q_{i}}, \frac{d \tau}{d t}=\sum_{j=1}^{n} p_{j} \frac{\partial H}{\partial p_{j}}
$$

Here $2 n$ component $q_{i}=q_{i}(t), p_{i}=p_{i}(t)$ which are solutions of the system (1.11) are called the characteristics of the equation (9).

If $\boldsymbol{q}_{\boldsymbol{i}}=\boldsymbol{x}_{\boldsymbol{i}}$ - the Cartesian coordinates, and $H=H(\bar{p}, \bar{r}), \bar{r}=\left(x_{1}, x_{2}, x_{3}\right)$, the Hamiltonian characteristic equations (1.11) - (1.13) can be written in the vector form

$$
\begin{gathered}
\frac{d \bar{r}}{d t}=\frac{\partial H}{\partial \bar{p}}, \frac{d \bar{p}}{d t}=-\frac{\partial H}{\partial r} \\
\frac{d \tau}{d t}=\bar{p} \frac{\partial H}{\partial \bar{p}}, \bar{p}=\operatorname{grad} \tau=\nabla \tau
\end{gathered}
$$

Differentiation respect to the vector, means differentiation with respect to the appropriate coordinate, for example $\frac{d x_{i}}{d t}=\frac{\partial H}{\partial \overline{p_{i}}}$. Thus, the ray is in the coordinate space of a projection $q_{i}=x_{i}=x_{i}(t)$ of the eikonal equation characteristics (1.4). In the phase space $\left\{p_{i}, q_{i}\right\}$ the characteristic $q_{i}=q_{i}(t) p_{i}=p_{i}(t)$ is called the ray equations.

If you found the solution of equations (1.13) in the form of $\bar{r}=r(t), \bar{p}=\bar{p}(t)$, then the solution of equation
(1.14) can be written as

$$
\tau=\tau_{0}+\int_{t_{0}}^{t} \bar{p} \frac{\partial H}{\partial \bar{p}} d t
$$

There are different forms of recording the ray equations (1.13), depending on the kind of $H$, coordinate system selection. For example, we write $H$ (eikonal equation) as

$$
H=\frac{1}{2}\left[\bar{p}^{2}-n^{2}(\bar{r})\right]=0, \bar{p}=\operatorname{grad} \tau
$$

where $n-$ the refractive medium index.

Then the equations for the rays have the form of

$$
\begin{gathered}
\frac{d \bar{r}}{d t}=\bar{p} \\
\frac{d \bar{p}}{d t}=\frac{1}{2} \nabla n^{2}(r)=\frac{1}{2} \operatorname{grad} n^{2}
\end{gathered}
$$

which shows that in an isotropic medium rays are orthogonal to the wave fronts.

Most convenient to use instead of the parameter $t$ the parameter of the arc lengths of the curved ray in a inhomogeneous medium

$$
d t=\frac{d s}{p}=\frac{d s}{n}
$$

then the expression for the eikonal (1.16) has the form

$$
\tau=\tau_{0}+\int_{s_{0}}^{s} n(\bar{r}) d s
$$

If the eikonal equation (and) or H-equation of Hamilton-Jacobi (1.5) will be written in the form of

$$
H(\bar{p}, \bar{r})=p-r(\bar{r})=0, ? p=\sqrt{\bar{p}^{2}}
$$

then the rays equations (1.14) have the form

$$
\frac{d \bar{r}}{d s}=\frac{\bar{p}}{n}, \frac{d \bar{p}}{d t}=\nabla n=\operatorname{grad} n
$$

and the expression for the eikonal (1.20).

To this same expression for the eikonal respond equations for the rays, written in the form

$$
\frac{d \bar{r}}{d s}=\bar{\ell}, \frac{d \bar{\ell}}{d s}=\frac{\operatorname{grad} n}{n}-\bar{\ell}\left(\frac{\operatorname{grad} n}{n} \bar{\ell}\right)=\operatorname{grad} \perp \ln n(1.23)
$$

where $\bar{\ell}^{*} \operatorname{grad} n=d r / d s-$ derivative with respect to the ray, the operator $\operatorname{grad}_{\perp}=\operatorname{grad}-\bar{\ell}(\bar{\ell}$ grad $)$ determines the gradient calculation in the direction perpendicular to the beam (along the wave front). 
According to the optical-mechanical analogy the system of equations (1.14) can also be written in the form of Newtonian mechanics for potential forces (in the form of second-order equations)

$$
\frac{d^{2} \bar{r}}{d t^{2}}=\frac{1}{2} \operatorname{grad} n^{2} \text { or } \frac{d}{d s}\left(n \frac{d \bar{r}}{d s}\right)=\operatorname{grad} n^{2}
$$

where the role of the forces potential is played by $n^{2}(\bar{r})$.

The geometry of the spatial curve (ray) is characterized by the curvature $\boldsymbol{k}$ and torsion $\varkappa$, which are calculated according to the formulas

$$
k=\left|\left[\bar{\ell}\left(\frac{\operatorname{grad} n}{n} \bar{\ell}\right)\right]\right|=\left|\left[\frac{\operatorname{grad} n}{n} \bar{\ell}\right]\right|=\left|\frac{\operatorname{grad} n}{n}\right|
$$

where $0<\theta<\frac{\pi}{2}-$ the angle between the ray (vector $\bar{\ell} \sin \theta)$ and vector $\operatorname{grad} n$.

The radius of the ray curvature $\rho=k^{-1}$. As in a homogeneous medium $n=$ const, then $k=0$, ie rays are straight lines.

The torsion is calculated according to the formula

$$
\varkappa=\frac{1}{2}(\bar{n} \operatorname{rot} \bar{n}+\bar{b} \operatorname{rot} \bar{b})
$$

where $\bar{n}-$ the unit vector the main normal, $\bar{b}=\left[\bar{\ell}^{*} n\right]$ the binormal unit vector in the Frenet trihedron, moving along the ray.

The expressions for $\bar{n}$ and $\bar{b}$ can be represented by the index of refraction $n(\bar{r})$ according to the formulas

$$
\bar{n}=\frac{1}{k} \operatorname{grad}_{\perp} \ln n, \bar{b}=\frac{1}{k}\left[\bar{\ell} \frac{\operatorname{gradn}}{n}\right]
$$

Then

$$
\begin{aligned}
& \varkappa=\frac{1}{k}\left[(\bar{\ell} \operatorname{grad}) \bar{b} * \operatorname{grad}_{\perp} \ln n\right]= \\
& =\frac{1}{k}\left[(\bar{\ell} \operatorname{grad}) \operatorname{grad}_{\perp} \ln n+\bar{b}\right]
\end{aligned}
$$

For planar curves $\varkappa=0$, for example in layered media.

Discussed equations correspond to coordinate method of setting a motion in mechanics. Natural way to set a motion in mechanics corresponds to the consideration of the kinematics of the rays in the ray coordinates, related to the initial position of the wave front $[4,7,15]$.

On the surface of the radiating body curvilinear coordinates can be introduced $\xi, \eta$ Fig. 1.6.

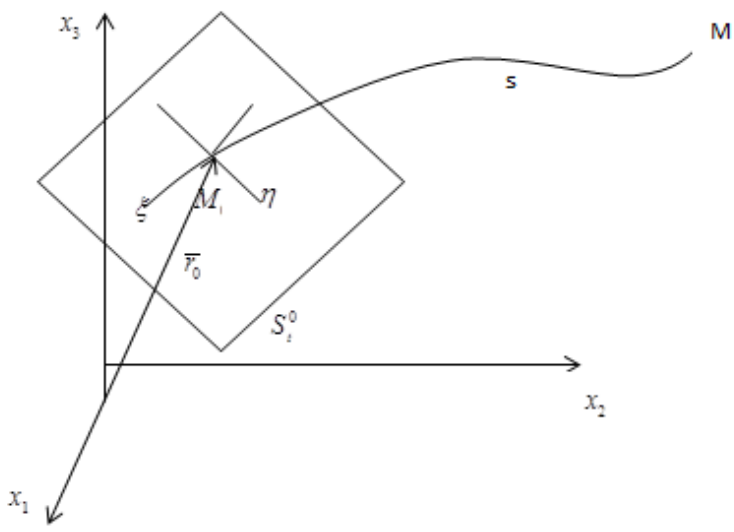

Figure 1.6. The ray coordinates

so that

$$
\overline{r_{0}}=\bar{r}\left(t_{0}\right)=\bar{r}_{0}(\xi, \eta)
$$

The coordinate lines $\xi, \eta$ are orthogonal and they are chosen, as a rule, from geodesic lines or lines of the principal curvatures, coordinate line $s$-ray, tangent to which at $\xi, \eta$ is orthogonal to the front $S_{t}^{0}$. Coordinate system $\xi, \eta, s$ is called as ray coordinate system.

At $t=t_{0}$ you must set a condition for $\bar{p}=\bar{p}\left(t_{0}\right)=\bar{p}_{0}(\xi, \eta)$. The vector components $\bar{p}(\xi, \eta)$ satisfy the equations, which follow from the eikonal equation.

$$
\begin{aligned}
& \left(\bar{p}^{0}\right)^{2}=n^{2}\left(\bar{r}_{0}\right), \bar{p}^{0} \frac{\partial \bar{r}^{0}}{\partial \xi}, \\
& \bar{p}^{0} \frac{\partial \bar{r}_{0}}{\partial \xi}=\frac{\partial \bar{\tau}_{0}}{\partial \xi}, \bar{p}^{0} \frac{\partial \bar{r}^{0}}{\partial \eta}=\frac{\partial \tau_{0}}{\partial \eta}
\end{aligned}
$$

If the initial emitting surface is plane, then the coordinate system is a Cartesian system, and is connected with the surface $x_{3}^{0}=0$. Then assuming that $\xi=x_{1}^{0}, \eta=x_{2}^{0}$, we obtain

$$
\begin{aligned}
& p_{x}^{0}=\frac{\partial \tau_{0}}{\partial \xi}, p_{y}^{0}=\frac{\partial \tau_{0}}{\partial \eta}, p_{z}^{0}= \\
& =\sqrt{n^{2}\left(\bar{r}_{0}\right)-\left(\frac{\partial \tau_{0}}{\partial \xi}\right)^{2}-\left(\frac{\partial \tau_{0}}{\partial \eta}\right)^{2}}
\end{aligned}
$$

The equation of the initial surface can be written as

$$
x_{i}=x_{i}\left(u^{\alpha}, t\right), \alpha=1,2 ; i=1,2,3
$$

We introduce the first and second quadratic form of the surface $\boldsymbol{S}_{\boldsymbol{t}}^{0}$ 


$$
g^{\alpha \beta}=x_{i}^{\alpha} * x_{i}^{\beta}, b^{\alpha \beta}=-v_{\alpha}^{i} * x_{\beta}^{i}=x_{\alpha \beta}^{i} v^{i}
$$

Then the differential equations for the rays have the form $[4,7,10]$

$$
\begin{gathered}
\frac{\delta x^{i}}{\delta t}=c v^{i}, c=v^{i} \frac{\delta x^{i}}{\delta t} \\
\frac{\delta v^{i}}{\delta t}=-g^{\alpha \beta} x_{\beta}^{i} v^{i} \frac{\delta x_{i}^{\alpha}}{\delta t}=-g^{\alpha \beta} x_{i \beta} c_{\alpha}
\end{gathered}
$$

To close the system (1.31), (1.32) it must be supplemented by equations for the mean and Gaussian curvatures of the surface.

In the particular case when the wave front propagates parallel to itself expressions for the mean and Gaussian curvatures have the form

$$
\Omega=\frac{\Omega_{0}-s K_{0}}{1-2 s \Omega_{0}+s^{2} K_{0}} K=\frac{K_{0}}{1+s^{2} K_{0}-2 s K_{0}}
$$

The expression for the eikonal in the coordinates $\xi, \eta, t$ is written in the form

$$
\tau(\xi, \eta, t)=\tau_{0}(\xi, \eta)+\int_{t_{0}}^{t} n^{2}[\bar{r}(\xi, \eta, t)] d t
$$

Coordinates $\xi, \eta$ on the surface $\tau_{0}$ identify a ray coming from the surface at the moment $t=t_{0}$. For different $\xi, \eta$ we obtain a family of rays, therefore, at the initial time from the surface $\tau_{0}$ comes the bundle of rays, that allows you to build a complex radiation pattern, on the basis of which is determined the wave field structure in the physical space.

In the phase space $\{\bar{p}, \bar{r}\}$ the phase portrait under certain conditions, can also be quite complicated.

In the spatial of generalized coordinates $\boldsymbol{q}_{\boldsymbol{i}}$, which are not related to the wave surface and which are orthogonal curvilinear coordinates, eikonal can be written as

$$
H\left(p_{i}, q_{i}\right)=\frac{1}{2} \sum_{i=1}^{3}\left\{\frac{p_{j}^{2}}{\mathrm{~h}_{j}^{2}\left(q_{i}\right)}-n^{2}\left(q_{i}\right)\right\}
$$

where $\mathbf{h}_{j}\left(\boldsymbol{q}_{\boldsymbol{i}}\right)$ - Lame coefficients for curvilinear coordinates $q_{i}$.

The system of equations for the rays in this case has the form

$$
\frac{d q_{i}}{d t}=\frac{1}{h_{i}^{2}} p_{i}, \frac{d p_{i}}{d t}=n \frac{\partial n}{\partial q_{i}}+\sum_{j=1}^{3} \frac{1}{h_{j}^{2}} \frac{\partial h_{j}}{\partial q_{i}} p_{j}^{2}
$$

The expression for the eikonal is written as

$$
\tau=\tau_{0}+\int_{\tau_{0}}^{\tau} n^{2}[\bar{r}(t)] d t, p^{2}=n^{2}
$$

If you enter a pulse components in curvilinear coordinates according to the formulas

$$
\hat{p}_{i}=\frac{P_{i}}{h_{i}}=\frac{1}{\mathrm{~h}_{i}} \frac{\partial \tau}{\partial q_{i}}, i=\overline{1, n}
$$

then the equations (1.36) are written as

$$
\frac{d \hat{p}_{i}}{d t}=\frac{1}{h_{i}} n \frac{\partial n}{\partial q_{i}}+\frac{1}{h_{i}} \sum_{j \neq i}^{3} \frac{\hat{p}_{j}}{h_{j}}\left(\hat{p}_{j} \frac{\partial h_{j}}{\partial q_{i}}-\hat{p}_{i} \frac{\partial h_{i}}{\partial q j}\right)
$$

In many specific problems of ray propagation in inhomogeneous media is convenient to use angular variables, for example, in the case of the spherical symmetry. Assuming that $q_{1}=r, q_{2}=\theta, q_{3}=\varphi$ and considering that in this case $\mathrm{h}_{1}=1, \mathrm{~h}_{2}=r, \mathrm{~h}_{3}=r \sin \theta$, equations (1.39) can be written as

$$
\begin{gathered}
\frac{d r}{d t}=\hat{p}_{r}, \frac{d \theta}{d t}=\frac{1}{r} \hat{p}_{\theta}, \frac{d \varphi}{d t}=\frac{\hat{p}_{\varphi}}{r \sin \varphi}, \\
\frac{d \hat{p}_{r}}{d t}=n \frac{\partial n}{\partial r}+\frac{1}{r} \hat{p}_{0}^{2}+\frac{1}{r} \hat{p}_{\varphi}^{2} \\
\frac{d \hat{p}_{\theta}}{d t}=\frac{1}{r}\left(n \frac{\partial n}{\partial \theta}-\hat{p}_{r} \hat{p}_{\theta}+c t g j \theta^{*} \hat{p}_{\varphi}^{2}\right) \\
\frac{d \hat{p}_{\varphi}}{d t}=\frac{1}{r \sin \theta}\left(n \frac{\partial n}{\partial \varphi}-\sin \theta \hat{p}_{r} \hat{p}_{\theta}-\cos \theta^{*} \hat{p}_{\theta} \hat{p}_{\varphi}\right) \\
\text { Here } \frac{\partial \tau}{\partial r}=\hat{p}_{r}, \frac{1}{r} \frac{\partial \tau}{\partial \theta}=\hat{p}_{\theta}, \frac{1}{r \sin \theta} \frac{\partial \tau}{\partial \theta}=\hat{p}_{\varphi}
\end{gathered}
$$

The eikonal equation is written as

$$
\left(\frac{\partial \tau}{\partial r}\right)^{2}+\left(\frac{1}{r} \frac{\partial \tau}{\partial \theta}\right)^{2}+\left(\frac{1}{r \sin \varphi} \frac{\partial \tau}{\partial \varphi}\right)^{2}=n^{2}
$$

Or

$$
\hat{p}_{r}^{2}+\hat{p}_{\theta}^{2}+\hat{p}_{\varphi}^{2}=n^{2}
$$

In the case of a plane task 2D-dimension, entering angle $\alpha$ by relations

$$
\begin{aligned}
& \hat{p}_{r}=n \cos \alpha \\
& \hat{p}_{\theta}=n \sin \alpha
\end{aligned}
$$

we write the equations for the ray in the form of

$$
\frac{d \theta}{d r}=\frac{1}{r} \operatorname{tg} \alpha, \frac{d \alpha}{d r}=\frac{1}{n r}\left[\frac{\partial n}{\partial r}-\frac{\partial(r n)}{\partial r} \frac{d \theta}{d r}\right] \alpha=\pi / 2
$$


Thus, depending on the geometry and mechanics of the particular task you can choose a suitable system of coordinates and shape of the ray equations. The most commonly used are phase coordinates $(\bar{r}, \bar{p})$ and radial coordinates $(\xi, \eta, s)$ so that the solution of the radial equation is represented as

$$
\bar{r}=\bar{r}(\xi, \eta, t), \bar{p}=\bar{p}(\xi, \eta, t)
$$

Here, the parameter $t$ It related to the distance $s$ along the ray (coordinate line), and $\xi, \eta$ identify the ray on the initial surface $S_{t}^{0}$ at $t=t_{0}$. If the wave is emitted by a limited surface area, defined by the relation $\bar{r}^{0}=\bar{r}^{0}(\xi, \eta)$, then the rays form a family of rays emanating from this area.

The conversion from the Cartesian coordinate system to the ray system is defined by Jacobian $D=\frac{\partial\left(x_{1}, x_{2}, x_{3}\right)}{\partial(\xi, \eta, t)}$ and will be one to one, if $\mathrm{D} \neq 0$.

A lot of wavefronts obtained in accordance with the principle of Huygens (contact transformations) form a family of equal phase surfaces, eikonal for each of them is written in the form

$$
\tau(\xi, \eta, t)=\tau^{0}(\xi, \eta)+\int_{t_{0}}^{t} n^{2}[r(\xi, \eta, t)] d t=\text { const }=\tau_{0}
$$

From the first equation (1.48) for each $\tau_{0}$ can be found $t=t_{\phi}(\xi, \eta, t)$ and it is inserted into the second, then the family of wave fronts of equal phase (phase fronts) is determined by the ratio

$$
\bar{r}=\bar{r}\left[\xi, \eta, t_{\phi}\left(\xi, \eta, \tau_{0}\right)\right]=r_{\phi}\left(\xi, \eta, \tau_{0}\right)
$$

The family of rays emitted by the limited surface area $S_{t}^{0}$ forms the bundle of rays. This means that the rays propagate not independent of each other, but they interfere. Due to the interference of secondary waves a significant contribution to the building of the fronts contribute only those rays, for which the phase difference does not differ by more than $\lambda / 2$ ( $\lambda$ - wavelength).

Surfaces, where condition $D_{\left(t_{K}\right) \mid S_{K}}=0$ is violated are referred to as caustic

$$
D_{\left(t_{K}\right) \mid S_{K}}=0 \mathrm{at} \bar{r} \epsilon \bar{r}_{k}(t)
$$

The position of caustics is defined from the equation of the family of rays $\bar{r}=\bar{r}(\xi, \eta, t)$ and condition

$$
\begin{array}{ll}
D(t)=0 & \\
& \quad \bar{r}=\bar{r}(\xi, \eta, t), D(\xi, \eta, t),=0
\end{array}
$$

Excluding $t$ we obtain

$$
\bar{r}=\bar{r}(\xi, \eta, t(\xi, \eta))=\overline{r_{k}}(\xi, \eta)
$$

where $\bar{r}_{k}(\xi, \eta)$ determines the equation of the caustic in curvilinear coordinates of the initial surface. In solving problems for caustics it is convenient to introduce on the caustic surface own caustic coordinates $(\alpha, \beta, \delta)$, where, $\alpha, \beta$ are curvilinear coordinates located on the caustic, and $\delta$ is measured along the line characterizing the removing from the caustic.

The value $J$ Excluding $t$ we obtain

$$
\begin{gathered}
J=\frac{D(t)}{D\left(t_{0}\right)}=\frac{\left(\left[\bar{r}_{, \xi} * \bar{r}_{, \eta}\right] * \bar{p}\right)}{\left(\left[\bar{r}_{, \xi} * \bar{r}_{, \eta}\right] * \bar{p}\right)_{t=t_{0}}} \\
\bar{r}_{, \xi}=\frac{\partial \bar{r}}{\partial \xi}, \bar{r}_{, \eta}=\frac{\partial \bar{r}}{\partial \eta}, \bar{r}_{, t}=\frac{\partial \bar{r}}{\partial t}=\bar{p}
\end{gathered}
$$

is called the divergence of rays.

From (1.51) follows that on the caustic $J=0$, ie cross-section of the ray tube decreases, energy increases, the rays touch caustics and change the direction. The classification of caustics is considered in the catastrophe theory [16]. On caustics and in their neighborhood classical spatial ray solutions are not applicable. There are methods for caustic rays, allowing to solve a number of tasks for caustics [2].

\section{Determinate Chaos of Rays and Fronts in Inhomogeneous Media with Deterministic Structure}

The equations describing the propagation of rays in inhomogeneous media are nonlinear because the refractive index $n(\bar{r})$ dependence on the spatial coordinates. The consequence is the possibility of deterministic chaos in the radiation pattern, when for a determinate particular dependence $n(\bar{r})$ is obtained not a concrete realization of the trajectory of the ray, but a set of possible trajectories, in the same way as is the case for random functions. The optical-mechanical analogy allows us to consider this problem with the most common positions within the Hamiltonian mechanics. Issues of construction of ray trajectories for different types of the determinate inhomogeneity are subject to numerous studies especially for 2D layered models. Significantly less works are devoted to the chaotization of ray trajectories in determinate inhomogeneous media [11-14].

First the possibility of deterministic chaos in inhomogeneous media has been considered in [13].

As is well known, the Poynting-Umov vector of energy 
density is directed along the trajectory of the ray along the tangent and thus chaos in the flows of energy and information in inhomogeneous media greatly complicates the prediction of information and energy processes.

In accordance with the optical-mechanical analogy of differential equations for the ray trajectories in inhomogeneous media are derived from Fermat's principle [1-4].

$$
\tau=\int_{M_{0}}^{M} n(\bar{r}) d s
$$

and in the Cartesian coordinate system can be written a

$$
\frac{d \bar{r}}{d s}=\bar{\ell}, \frac{d}{d s}\left(n(\bar{r}) \frac{d \bar{r}}{d s}\right)=\nabla \tau(r)=\operatorname{grad} \tau
$$

The wave surface is determined by the eikonal equation

$$
\frac{d \tau}{d s}=n(\bar{r})
$$

where $\bar{r}=\bar{r}\left(x_{1}, x_{2}, x_{3}\right), \xi-$ the distance along the ray trajectory.

The equation (2.2) can be written in the Hamiltonian form, if we assume that the Hamiltonian $H$ has the form of

$$
H=-\sqrt{n^{2}-p^{2}}
$$

where $\bar{p}=\left(p_{1}, p_{2}, p_{3}\right), p=|p|=\sqrt{p_{1}^{2}+p_{2}^{2}+p_{3}^{2}}$.

Then, dividing the spatial coordinates $\left(x_{1}, x_{2}, x_{3}\right)=\bar{r}$, for example, $x_{1}, x_{2}$ we take as coordinates on a locally plane wave, and $x_{3}$ directed along the straight ray orthogonal to the plane $0 x_{1}, x_{2}$ Fig. 2.1.

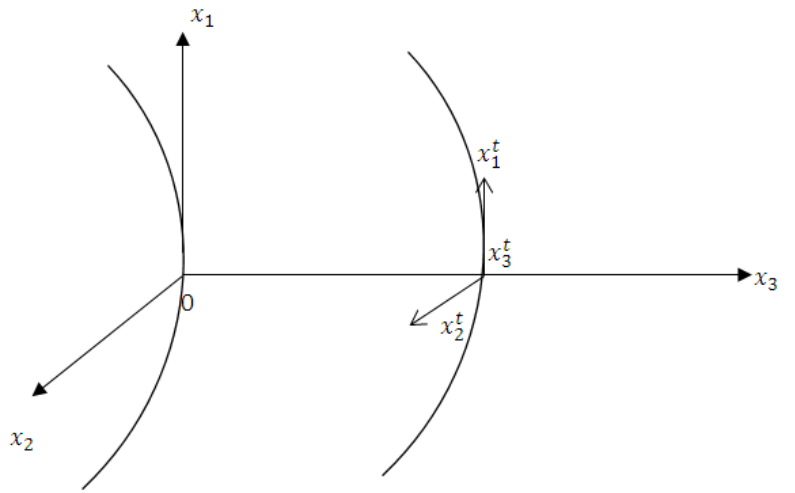

Figure 2.1. The spatial coordinates on a locale plane wave at the time $t=0$ at the moment $t$

Then imagine $\bar{r}=\left(x_{1}, x_{2}, x_{3}\right)=\left(\bar{\rho}, x_{3}\right), \bar{\rho}=\left(x_{1}, x_{2}\right)$, where $\bar{\rho}$ a function of is $x_{3}$ and determines the coordinates of the ray point in the tangent plane of the wave front.

Impulse $\bar{p}$ is introduced by the relation

$$
\bar{p}=\frac{n \bar{p}}{\sqrt{1-p^{2}}}, \bar{p}=\frac{d \bar{p}}{d x_{3}}
$$

Taking into account (2.4), (2.5) the Hamiltonian form of the equations for the ray has the form of

$$
\frac{d \bar{p}}{d x_{3}}=-\frac{\partial H}{\partial \bar{p}}, \frac{d \bar{p}}{d x_{3}}=\frac{\partial H}{\partial \bar{p}}
$$

The waveguide in a inhomogeneous medium is a linearly extended volume, where takes place a determinate expressed change of the refractive index from its boundaries to the axis in such way, that rays falling or occurring therein are propagating along its axis in general experiencing fluctuations near the waveguide axis, waveguide may be inhomogeneous along the waveguide.

The axis of the waveguide $\mathrm{Ox}_{3}$ is the perpendicular to the plane $\mathrm{Ox}, x_{2}$ and an attractor for ray trajectories, if the change $n$ along $x_{1}, x_{2}$ has the character of monotonic increase with a maximum on the axis $\mathrm{Ox}_{3}$ Fig. 2.2.



Figure 2.2. The dependence $n(\rho)$ in a cross-section of the waveguide

In this case rays emitted in the waveguide parallel to the axis $O x_{3}$ and rays, entering into the waveguide at some angles oscillate near axis $x_{3}$. The ray acts like a ball, received an initial impulse moving along the ideal (frictionless) chute along the axis $x_{3}$ (in the plane $O x, x_{2}$ is a potential well).

Accounting for the presence of a certain type of inhomogeneity along the axis $O x_{3}$ may result in a deterministic chaos in radiation pattern.

We represent the refractive index $n^{2}\left(\bar{\rho}, x_{3}\right) \rho=\sqrt{x_{1}^{2}+x_{2}^{2}}$ as 


$$
n^{2}\left(\bar{\rho}, x_{3}\right)=n^{2}(\bar{\rho})+\mathcal{V}\left(\bar{\rho}, x_{3}\right)
$$

Here $n^{2}(\bar{\rho})$ determines the refractive index in the waveguide excluding inhomogeneity in the direction $x_{3}$, and $\mathcal{V}\left(\bar{\rho}, x_{3}\right)$ takes into account the presence of inhomogeneity along the waveguide $x_{3}, \varepsilon \ll 1-$ a small dimensionless parameter.

In accordance with the representation (2.7) we can, using classical perturbation theory to write down [1-4, 11-14]

$$
H=H_{0}(\bar{\rho}, \bar{p})+\varepsilon H_{1}\left(\bar{p}, \bar{\rho}, x_{3}\right)
$$

where the summands $H_{0}, H_{1}$ have the form of

$$
\begin{aligned}
H_{0}(\bar{\rho}, \bar{p}) & =-\sqrt{n_{(\bar{\rho})}^{2}-p^{2}} \\
H_{1}\left(\bar{\rho}, \bar{p}, x_{3}\right) & =\mathcal{V}\left(\bar{\rho}, x_{3}\right) H_{0}^{-1}
\end{aligned}
$$

The wave nature of propagation of the rays takes place in the ocean acoustics, geophysics, where the free surface of the Earth has waveguide properties and that provides further propagation of surface waves, in optics, in particular, the propagation of light through fiber optic wires.

We assume that the quasi-cylindrical waveguide has a axially symmetrical nature of the change of the refractive index $n(\bar{\rho})$, then at any section of the waveguide along the axis $\mathrm{Ox}_{3}$ the ray pattern will be the same, so for simplicity we consider the case of $2 \mathrm{D}$, when $n(\bar{\rho})=n\left(x_{1}\right)$.

Equations (2.8) - (2.10) and therefore (2.6) have the form of

$$
\begin{gathered}
H=H_{0}+\varepsilon H_{1}\left(x_{1}, p, x_{3}\right) \\
H_{0}=-\sqrt{n^{2}\left(x_{1}\right)-p^{2}} \\
H_{1}=\mathcal{V}\left(x_{1}, x_{3}\right) H_{0}^{-1} \\
\frac{d p}{d x_{3}}=-\frac{\partial H}{\partial x_{1}}, \frac{d x_{1}}{d x_{3}}=\frac{\partial H}{\partial p}, p=p_{1}
\end{gathered}
$$

In the phase plane $x_{1}, p_{1}$ phase trajectories of the ray have the form corresponding to the center

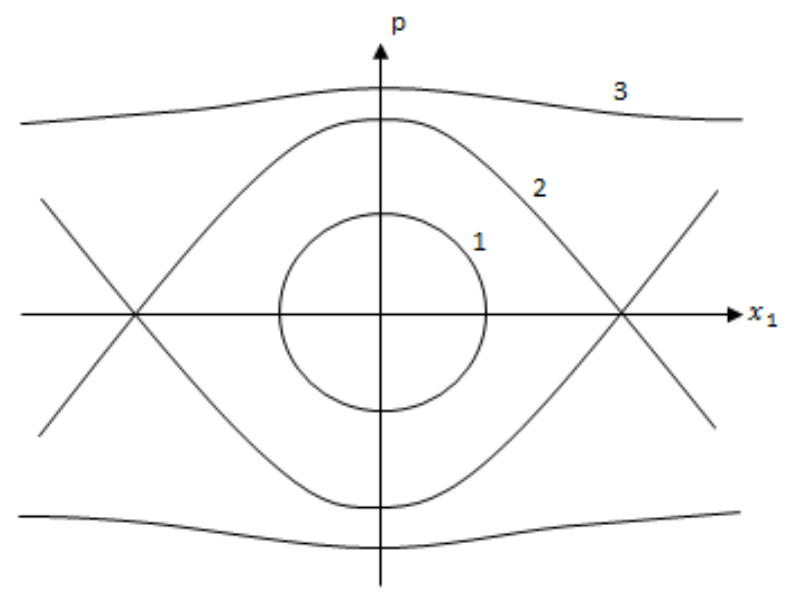

Figure 2.3. Phase trajectories of the rays in the waveguide

We proceed to the action-angle variables $(I, \theta)$

$$
\begin{gathered}
I=\frac{1}{2 \pi} \int p_{1} d x_{1}, \theta=\frac{\partial S\left(x_{1}, I\right)}{\partial I} ? \\
S\left(x_{1}, I\right)=\int_{0}^{x_{1}} p d x_{1}, p=\sqrt{n^{2}\left(x_{1}\right)-E^{2}}
\end{gathered}
$$

which are associated with the wave front energy $E$ along the ray by relation

$$
\frac{d E(I)}{d I}=\omega(I), \theta=\omega t+\varphi
$$

where $\omega(I)$ - frequency.

Writing the Hamiltonian and equations ray trajectories in variables $I, \theta$, we obtain

$$
\begin{gathered}
H\left(I, \theta, x_{3}\right)=H_{0}(I)+\varepsilon H_{1}\left(I, \theta, x_{3}\right) \\
\dot{I}=-\varepsilon \frac{\partial H_{1}}{\partial \theta}, \dot{\theta}=\omega(I)+\varepsilon \frac{\partial H_{1}}{\partial I}
\end{gathered}
$$

Equations (2.18) and (2.19) describe nonlinear oscillations of the ray that causes anisotropy of its oscillations, i.e. the frequency of the ray oscillation near the axis of the waveguide depends on its energy.

Parameter $\alpha$, defined by the formula

$$
\alpha=\left|\frac{I}{\omega} * \frac{d \omega}{d I}\right|=2\left|\frac{\partial^{2} H_{0}}{\partial^{2} I} / \frac{\partial H_{0}}{\partial(I)^{2}}\right|
$$

characterizes the degree of system nonlinearity. 
Presenting the disturbance $H_{1}$ in the form of a double Fourier series

$$
H_{1}\left(I, \theta, x_{3}\right)=\sum_{m, n} \frac{H_{m n}^{(1)}(I)}{2} e^{i(m \Omega+n \theta)}
$$

The condition for the occurrence of nonlinear resonance in the ray vibrations can be written as

$$
m \Omega+n \omega \approx 0, ? \dot{\sigma}=\Omega
$$

where $\Omega=T / 2 \pi$ - frequency, and $T$ - the period of disturbances of nonuniformity along the axis of the waveguide $x_{3}, \sigma$-phase of vibrations' disturbances.

The closeness of the relation (2.22) to zero depends on the width of the resonance.

For a single resonance $m, n= \pm 1(\omega \approx \Omega)$ sum in (2.21) has two real summands: with the argument $\dot{\sigma}-\theta$ (resonance summand) and with the argument with the argument $\dot{\sigma}+\theta$ (high-frequency disturbance). As is known, if the localized resonances are far enough from each other, then leaving in (2.21) only the resonant summand we obtain

$$
\begin{gathered}
\dot{I}=\frac{1}{2} \varepsilon H_{m n}^{(1)} \sin \psi_{m n} \\
\dot{\psi}_{m n}=m \Omega+n \omega(I)+\frac{1}{2} \varepsilon n H_{m n, I}^{(1)} \cos \psi_{m n}
\end{gathered}
$$

where $\psi_{m n}=m \dot{\sigma}+n \theta-$ resonance phase, comma denotes differentiation with respect to the action $I$.

In the case of moderate nonlinearity characterized with the parameter $\varepsilon \ll \alpha \ll \varepsilon^{-1}$, difference $\Delta I=I-I_{p}$ is small $\left(|\Delta I| \ll I_{p}\right)$, where $I_{p}-$ then the value of $I$ in which we have the exactly relation (2.22) $m \Omega+n \omega\left(I_{p}\right)=0$.

Equations (2.23) correspond to the Hamiltonian

$$
H_{A}=m \omega_{, I} \frac{(\Delta I)^{2}}{2}+\varepsilon n H_{m n}^{(1)}\left(I_{p}\right) \cos \psi_{m n} ; \omega_{, I}=\frac{d \omega}{d I \mid I=I_{p}}
$$

From (2.24) we obtain the maximum width of the nonlinear resonance

$$
\begin{gathered}
(\Delta I)=4 \sqrt{\varepsilon\left(\omega_{, I}\right)^{-1} H_{m n}^{(1)}} \\
(\Delta \omega)=\left(\omega_{, I}\right)(\Delta I)=4 \sqrt{\varepsilon \omega_{, I} H_{m n}^{(1)}}=4 n^{-1} \Omega_{m n}
\end{gathered}
$$

where $\Omega_{m n}$ - the frequency of small phase oscillations.

The condition of moderate nonlinearity $\alpha$ means little value changes Iand $\omega$

$$
\frac{\Delta I}{I}=C\left(\frac{\varepsilon}{\alpha}\right)^{1 / 2}, \stackrel{\Delta}{,} \omega
$$

or approximately

$$
\frac{\Delta I}{I} \sim\left(\frac{\varepsilon}{\alpha}\right)^{1 / 2}, \frac{\Delta \omega}{\omega} \sim(\varepsilon \alpha)^{1 / 2}
$$

Due to the nonlinearity, fluctuations are nonisochronism, frequency depends on the amplitude. As a result of the changes in the amplitude of oscillations at a resonance frequency change and leaving its resonance value occur, there is termination of amplitude changes, causing the return of the oscillation frequency to a resonant value. This process is repeated, i.e. nonlinearity stabilizes isolated resonance, making the amount of change to be limited $\Delta I$.

For the moderate nonlinearity $\alpha \sim 1$ the decomposition is carried out by $\varepsilon^{1 / 2}$, indicating according to (2.28), that in the case of resonance $\Delta I \sim \varepsilon^{1 / 2}$, i.e. more than in a non-resonance case.

The condition of stabilization of nonlinear resonances has the form of

$$
\begin{gathered}
\varepsilon n H_{m n, I}^{(1)} \leq n(\Delta \omega) \text { or } \\
\varepsilon \alpha^{-1} \leq 16 \omega H_{m n, I}^{(1)} I^{-1}\left(H_{m n, I}\right)^{-2}
\end{gathered}
$$

Consider now the case when the resonances strongly interact with each other, the consequence of which is a stochastic instability of the ray oscillations. Resonances connection coefficient is introduced by the formula

$$
s=(\Delta \omega) \Delta^{-1}
$$

where $(\Delta \omega)$ is defined by the formula (2.27) and represents the width of the resonance, $\Delta=\left|\omega_{i+1}-\omega_{i}\right|-$ the distance from this resonance to the nearest neighboring resonance.

At $s \ll 1$ we obtain an isolated resonance case, at $s \gg 1$ resonances overlap, resulting in greatly irregular movement. Value $s \sim 1$ is the boundary of stochasticity or the criterion of instability.

For an arbitrary determinate periodic perturbation $H_{1}$ in the phase space of the neighborhood of the separatrix forms a layer, where ray trajectories are behaving chaotically. The width of the layer is determined by the specific form of a periodic perturbation.

Let disturbance have the form of

$$
H_{1}(I, \theta, \sigma)=T H_{1}(\theta) \sum_{K} \delta\left(x_{3}-k T\right)
$$

Using the canonical transformation $I \rightarrow \tilde{I}, \theta \rightarrow \tilde{\theta}$ we write the equations of motion in the form 


$$
\begin{gathered}
\dot{I}=\dot{I}-\varepsilon T \frac{\partial H_{1}}{\partial \theta}=\dot{I}-\varepsilon I_{0} f(\theta) \\
\dot{\theta}=\dot{\theta}+T \omega(I)
\end{gathered}
$$

where $f(\theta) \sim 1-$ dimensionless phase function, $I_{0}-$ constantly acting.

The transformation (2.32) describes the motion of the ray between the "points" of the inhomogeneity along the axis $x_{3}$ on the length interval $T$. At $T \rightarrow 0$, $\frac{\partial H_{1}}{\partial \theta}=0$ and then we obtain the canonical equations (2.6).

If the perturbation has one harmonic $f(\theta)=\sin \theta$, the resonance condition is written as: $T \omega=2 \pi m$, where $m$-whole number.

The system of resonances corresponds to the Fourier series expansion of the periodic $\delta$-function

$$
\sum_{K} \delta\left(x_{3}-k T\right)=\frac{1}{T} \sum_{m=-\infty}^{\infty} e^{i m \Omega x_{3}}
$$

where the distance between resonances is: $\Delta=2 \pi / T=\Omega, a H_{m_{1}}^{(1)}=2 I_{0} / T$.

Let the variables $I, \sigma$ of the ray trajectory in action-angle variables satisfy equations of the form

$$
\begin{gathered}
\dot{I}=\varepsilon I \sum_{k=-\infty}^{\infty} \delta\left(x_{3}-k T\right) \sin \sigma=\varepsilon H_{1}\left(I, \sigma, x_{3}\right) \\
\dot{\sigma}=\omega(I)
\end{gathered}
$$

The condition of conservation of phase volume has the form of $[16,17]$

$$
\frac{\partial f}{\partial x_{3}}+\omega \frac{\partial f}{\partial \sigma}+\varepsilon \frac{\partial}{\partial I}\left(H_{1} f\right)=0
$$

Then laying out $f$ and $H_{1}$ in the Fourier series

$$
\begin{gathered}
f\left(I, \sigma, x_{3}\right)=\sum_{h=-\infty}^{\infty} f_{n}\left(I, x_{3}\right) e^{i n \sigma}, n= \pm 1 \\
H_{1}\left(I, \sigma, x_{3}\right)=\sum_{h, k=-\infty}^{\infty} H_{n k}^{(1)}(I) e^{i\left(n \sigma+k \Omega x_{3}\right)}, \Omega=2 \pi / T
\end{gathered}
$$

and substituting (2.36) into (2.35), we obtain

$$
\frac{\partial f_{n}}{\partial x_{3}}+i n \omega f_{n}=-\varepsilon \frac{\partial}{\partial I} \sum_{s, k} H_{s k}^{(1)} * f_{n-s} e^{i k \Omega x_{3}}
$$

Consider the estimate of the probability of occurrence of deterministic chaos in radiation pattern. Imagine a discretization along the axis $x_{3}$ in the form of $x_{3}=N T(N \gg 1)$, then the equation (2.34) can be written as

$$
\begin{gathered}
I_{m+1}=I_{m}+\varepsilon I_{m} \sin \sigma_{m} \\
\sigma_{m+1} \approx \sigma_{m}+\omega T+K_{m} \sin \sigma_{m}, K_{m}=\frac{\varepsilon I_{m}}{\Omega} \frac{d \omega\left(I_{m}\right)}{d I_{m}}
\end{gathered}
$$

The correlation function of the phase has the form of

$$
R\left(x_{3}\right)=R_{N}=\frac{1}{2 \pi} \int_{0}^{2 \pi} d \sigma_{0} e^{i\left(\sigma_{N}-\sigma_{0}\right)}
$$

At $K \gg 1, R\left(x_{3}\right) \sim e^{\ln K-\Omega x_{3}+i \omega x_{3}}$.

It is believed ergodicity conditions are met.

We introduce the distribution function $F=(2 \pi)^{-1} \int_{0}^{2 \pi} f_{0} d \sigma_{0}, f_{0}-$ density.

At $K \gg 1$ the function $F$ satisfies the diffusion equation

$$
\frac{\partial F}{\partial x_{3}}=2 \pi \varepsilon^{2} \frac{\partial}{\partial I} \sum_{n, k}\left|H_{n k}^{(1)}\right| \delta(n \omega-k \Omega) \frac{\partial}{\partial I}\left|H_{n k}^{(1)}\right| F
$$

where $x_{3} \gg T$.

In this case the equation (2.40) describes ray diffusion (deterministic chaos)

At $K \ll 1$ averaging over $\sigma_{0}$ gives an estimate

$$
\frac{\partial F}{\partial x_{3}}=2 \pi \varepsilon^{2} \frac{\partial}{\partial I} \sum_{n, k}\left|H_{n k}^{(1)}\right| \delta(n \omega-k \Omega) \frac{\partial}{\partial I}\left|H_{n k}^{(1)}\right| F
$$

which shows that no randomization occurs, because correlation between points of the ray does not decrease.

Coefficient $K(\theta)$ has the form of

$$
K(\theta)=\frac{d \tilde{\theta}}{d \theta}-1=-\varepsilon \omega_{, I} I_{0} T f_{, 0}^{(0)}
$$

with $K(\theta) \sim s^{2}$.

Stochastization of rays at $K \gg 1$, characterized by the correlation function (2.39) is similar to processes such as pitching of the ship, movements of various gyroscopic systems, in electrical circuits of the simplest form under the influence of periodic actions.

As is well known, in irregular fiber waveguides of the gradient, refractive type, underwater, underground acoustic waveguides are possible phenomenon of rays' swing, swinging of the waveguide channel width, "rashes" of rays from the waveguide [2].

In [11] it was found a decrease in the effective cross waveguide size and flashing rays from it. 


\section{Expansion of Rays and Fronts in Stochastically Inhomogeneous Media}

Models of randomly inhomogeneous media allow to take into account the fact, that in real-world environments, in principle, it is impossible to determine (measure) accurately, to know the material physical and mechanical properties (coefficients) of the medium in sutu, because there is always the effect of artifacts related to measurements. Due to this experimental data always have a dispersion (scatter) in each point of the medium, i.e. can be modeled as a random field of physical media factors. Accordingly, rays and fronts will represent random trajectories and surfaces [16].

The equations for the rays and the eikonal in general case of an arbitrary dependence of the refractive index on the spatial coordinates cannot be solved analytically in the general case.

The most widely used is the method of successive approximations (a small parameter). According to the method analytical approximations for the coordinates ray and the eikonal sequentially are found, and then calculated numerically.

Imagine the square of the refractive index in the form of

$$
\begin{aligned}
n^{2}(\bar{r})= & n^{2}(\bar{r})+\mathcal{V}(\bar{r}) \text { or } \varepsilon(\bar{r})=\varepsilon(\bar{r})+\mathcal{V}(\bar{r}) \\
& \text { with } \max |\mathcal{V}(\bar{r})| \ll \varepsilon(\bar{r})
\end{aligned}
$$

In the submission

$\varepsilon(\bar{r})=n^{2}(r)$

unrecovered value of the refractive index, or the average $\mathcal{V}(\bar{r})$ - the fluctuation of the refractive index which takes into account the inhomogeneity of media.

Accordingly, submission (3.1) of an expression for the coordinates of the ray, the eikonal in a inhomogeneous medium are represented in the Cartesian coordinate system in the form of

$$
\begin{gathered}
\bar{r}(t)=\sum_{K=0}^{\infty} \bar{r}_{k}(t) \\
\tau(\bar{r})=\sum_{K=0}^{\infty} \tau_{k}(\bar{r})
\end{gathered}
$$

where $\bar{r}_{0}(t), \tau_{0}(\bar{r})$ - unperturbed (middle) trajectories of the ray and the eikonal, and $\bar{r}_{K}(t), z_{K}(\bar{r})(k \geq)$ give the corrections arising from the inhomogeneity.

Substituting (3.2) into equations (1.24), we obtain a recurrent system of ordinary differential equations

$$
\begin{gathered}
\bar{r}_{0}=\frac{1}{2} \nabla \varepsilon\left(r_{0}\right) \\
\bar{r}_{n}=\frac{1}{2}\left(r_{n} \nabla\right) \nabla \varepsilon\left(r_{0}\right)+\bar{F}_{n}, n=1,2 \ldots
\end{gathered}
$$

where $F_{n}$ depend only on prior approximations i.e. they are known functions.

Substituting (3.3) into equation (1.4), we obtain a recurrent system of differential equations in partial derivatives of the first order to find the corrections to the eikonal

$$
\begin{gathered}
(\nabla \tau)^{2}=\varepsilon(\bar{r}) \\
2\left(\nabla \tau_{0}, \nabla \tau_{1}\right)=\mathcal{V}(\bar{r}) \\
2\left(\nabla \tau_{0}, \nabla \tau_{n}\right)=-\left(\nabla \tau_{n-1}\right)^{2}, n=2, \ldots
\end{gathered}
$$

Consider two cases: 1. The medium is quasihomogeneous, then $\varepsilon=$ const and the rays will be in average straight

$$
\begin{gathered}
\bar{r}_{0}(t)=\bar{r}^{(o)}+\bar{p}^{(o)} t \tau=\tau^{(o)}+\varepsilon t \\
\bar{p}_{1}=\bar{r}_{1}=\bar{p}_{1}^{(0)}+\int \bar{F}_{1}\left(t^{1}\right) d t^{1} \\
\bar{r}_{1}=\bar{r}_{1}^{(0)}+t \bar{p}_{1}^{(0)}+\int_{0}^{t} d t^{t^{1}} \int_{0}^{1} F_{1}\left(t^{2}\right) d t^{2}=\bar{r}_{1}^{(0)}+ \\
+t \bar{p}_{1}^{(0)}+\int_{0}^{t}\left(t-t^{1}\right) \bar{F}_{1}\left(\tau^{1}\right) d t^{1}
\end{gathered}
$$

Here the initial values $\bar{r}^{(o)}, \bar{p}_{1}^{(0)}$ are selected at the initial wave surface. Assuming that $t_{0}=0, \bar{r}^{(0)}=0, \bar{p}_{1}^{(0)}=\mathcal{V}^{(0)} \bar{N} / 2 p_{N}^{(0)},(\bar{N}-$ normal to the primary surface $Q, \mathcal{V}^{(0)}$ refractive index at $\mathrm{Q}$, $\bar{p}_{N}^{(0)}$ - normal ray exit speed on $Q$ ), we obtain the trajectory of the ray in parametric form.

If within the first approximation in the solution found

$$
\bar{r}(t)=\bar{r}_{0}(\xi, \eta, s)+\bar{r}_{1}(\xi, \eta, s)
$$

To go to the ray coordinates $\xi, \eta, s$, then the inverse task is formulated: along the ray at the observation point to restore the coordinates of the ray in the point of emission $(\xi, \eta, s)$. In the zero-order approximation a ray is straight, the task is correct, taking into account the fluctuations of the inhomogeneity the system of equations (3.12) may have several roots, which corresponds to the arrival to the observation point a few rays, i.e. the presence of caustics in the rays' arrival point.

Consider the account of the eikonal corrections due to the refractive index fluctuations in two cases with and without taking into account the lateral displacement of the ray.

In the first case, considering that the solution of equation (3.7) for the unperturbed eikonal is unknown. Then, taking into account $\nabla \tau_{0}=\bar{p}=d \bar{r}_{0} / d t$, the 
equation (3.7) can be written as

$$
2\left(\nabla \tau_{0} \nabla \tau_{x}\right)=2 \frac{d \tau_{1}}{d t}=v
$$

Integrating (3.13) along the unperturbed ray we write

$$
\tau_{1}=\frac{1}{2} \int_{t_{0}}^{t} v d t^{1}=\frac{1}{2} \int_{s_{0}}^{s} \frac{v}{\sqrt{\varepsilon}} d s
$$

Accordingly, from (1.8) it can be obtained at $n=2$

$$
\tau_{2}=-\frac{1}{2} \int_{t_{0}}^{t}\left(\nabla \tau_{1}\right)^{2} d t^{1}=-\frac{1}{2} \int_{s_{0}}^{s} \frac{\left(\nabla \tau_{1}\right)^{2}}{\sqrt{\varepsilon}} d s
$$

These corrections are applicable, if the displacement corrections of the perturbed from the unperturbed ray are small compared with the characteristic cross-sectional dimension of the perturbation $\ell_{\perp}$.

Consider the account of the transverse ray displacement, which is determined by the solution of equation (3.5). The solution of equation (3.6) in this case has the form

$$
\tau=\tau^{(0)}+\int_{t_{0}}^{t}\left\{\begin{array}{l}
\varepsilon\left[r_{0}\left(t^{1}\right)+r_{1}\left(t^{1}\right)\right]+ \\
v\left[r_{0}\left(t^{1}\right)+r_{1}\left(t^{1}\right)\right]
\end{array}\right\} d t_{1}
$$

As follows from (3.16) an approximation to (3.14) is obtained from (3.16) by neglecting the transverse displacements $r_{1}$. This corresponds to the condition $\left|\bar{r}_{1}\right| \ll \ell_{\perp}$.

In case the medium is not quasi-homogeneous $n(\bar{r}) \neq$ const, the method of variation of arbitrary constants is applied which we use in mechanics to solve equations with variable coefficients [1]. Solution of the equations (1.5) at $k=1$, is represented as

$$
\bar{r}_{1}(t)=\sum_{k=1}^{6} c_{k}(t) \bar{\rho}_{k}(t)=\sum_{k=1}^{6} c_{k}(t) \frac{\partial \bar{r}_{0}(t)}{\partial \alpha_{k}}
$$

where $c_{k}(t)$ are coefficients that are required to determine, $\bar{\rho}_{k}(t)$ - the fundamental solutions of the system of uniform linear equations.

$$
\bar{r}_{k}=\frac{1}{2}\left(\bar{\rho}_{k} \nabla\right) \nabla \varepsilon\left(\bar{r}_{0}\right), k=\overline{1,6}
$$

The equation of the trajectory for a particular ray $\bar{r}_{0}(t)$ depends on the 6 initial conditions

$$
\begin{aligned}
& {\overline{r_{0}}}^{(0)}=\bar{r}_{0}(0)=\bar{\alpha}_{k},(k=1,2,3) ; \\
& \bar{\rho}_{0}^{(o)}=\bar{\rho}_{0}(0)=\bar{\alpha}_{k},(k=4,5,6)
\end{aligned}
$$

and the vector components $\bar{\rho}$ are determined by the formulas

$$
\begin{gathered}
\bar{\rho}_{k}(t)=\partial \bar{r}_{0}(t) / \partial \alpha_{k} \\
\sum_{k=1}^{6} \dot{c}_{k}(t) \bar{\rho}_{k}(t)=0 \\
\sum_{k=1}^{6} \dot{c}_{k}(t) \bar{\rho}_{k}(t)=\bar{F}_{1}=\frac{1}{2} \nabla v\left(\bar{r}_{0}\right)
\end{gathered}
$$

The solution of the system (3.21) is written as

$$
c_{k}(t)=c_{k}(0)+\sum_{j=1}^{3} \int_{0}^{t} Q_{k j}\left(t^{1}\right) F_{1 j}\left(t^{1}\right) d t^{1}
$$

where $c_{k}(0)$ are found from the initial conditions.

Substituting (3.22) in (3.17) we obtain expressions for ray correction in inhomogeneous media.

Consider the vector $\bar{p}=\bar{r}$, which can be represented as a series of

$$
\bar{p}(t)=\sum_{k=0}^{\infty} \bar{p}_{k}(t)=\sum_{k=0}^{\infty} \bar{r}_{k}(t)
$$

From (3.17) with regard to the first system in (3.21) it is obtained

$$
\bar{p}_{1}=\frac{d}{d t} \sum_{k=1}^{6} c_{k} \bar{p}_{k}=\sum_{k=1}^{6} c_{k} \bar{p}_{k}
$$

Through $\bar{p}_{1}$ correction $\bar{\ell}_{1}$ is calculated the unperturbed unit vector $\bar{\ell}_{0}$ - tangent to the undisturbed ray $\overline{r_{0}}$

$$
\bar{\ell}_{1}=\frac{\left(\bar{p}_{1}-\bar{\ell}_{0}\left(\bar{\ell}_{0} \bar{p}_{1}\right)\right)}{\sqrt{\varepsilon}}=\frac{\bar{p}_{\perp}}{\sqrt{\varepsilon}}
$$

Vector $\bar{\ell}_{1}$ characterizes the deflection angles of the perturbed ray from the unperturbed ray.

Use of the method of successive approximations (small parameter) is based on the presence of small parameters in the task about the wave propagation in a inhomogeneous medium: 1) the wavelength is much smaller than the scale of inhomogeneity changes (asymptotic behavior of the short waves - ray theory); 2) the maximum amplitude fluctuations of physical and mechanical parameters of the medium from their average values are small (weakly inhomogeneous media). 
We consider some of the effects of ray propagation in randomly inhomogeneous media within these approximations.

Then in the representation (3.1) the index of refraction $n(\bar{r})$ is a random function of the spatial coordinates (random field), which is described by its moments or probability distribution. Since in this case, the coordinate points of the trajectories of rays and eikonals will be random functions, then they are also described by the moments or probability distribution. We consider the momentary way of describing random functions within the correlation approximation, if $\sigma_{n} \ll \varepsilon$, where $\sigma_{n}$ is the mean square deviation of the refractive index, $\varepsilon$ is the mean square value of the refractive index. The average value and the correlation function of the refractive index $R_{n}\left(\bar{r}_{1}, \bar{r}_{2}\right)=\overline{\mathcal{V}}\left(\overline{r_{1}}\right) \overline{\mathcal{V}}\left(\bar{r}_{2}\right)$ are considered to be given. It is required to find the coordinates of points of the average ray trajectory, the average deviation of rays from the average the trajectory.

In the case where the initial emitting surface is plane and the initial emitted wave is plane too, will take the Cartesian coordinate system at the initial plane $S_{t}^{0}$ as shown in Figure 3.1

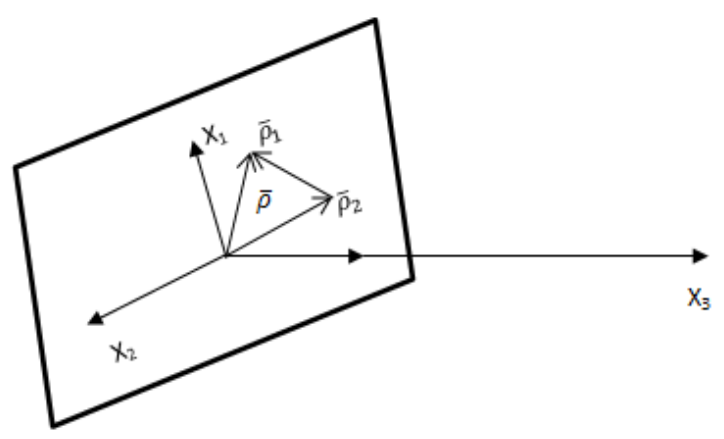

Figure 3.1. The initial emitting surface is plane

Axis $x_{3}$ is a straight ray in an inhomogeneous medium (trajectory of the middle ray in inhomogeneous medium).

Then, the average value of the eikonal $\tau_{0}=\sqrt{\varepsilon} x_{3}$, and the first approximation is equal to

$$
\tau_{1}=\frac{1}{2 \sqrt{\varepsilon}} \int_{0}^{x_{3}} \mathcal{V}\left(x_{1}, x_{2}, x_{3}^{1}\right) d x_{3}^{1}
$$

Considering that

$$
\sigma_{n} \ll \varepsilon
$$

the second-order approximation can be neglected.

The correlation function $R_{\tau}$ of the eikonal $\tau$ has the form

$$
R_{\tau}\left(\overline{r_{1}}, \bar{r}_{2}\right)=\frac{x_{3 \min }}{2(\sqrt{\varepsilon})^{2}} \int_{0}^{\infty} R_{n}(\bar{\rho}, \xi) d \xi
$$

Here $\bar{r}=\left(\bar{\rho}, x_{3}\right), \bar{\rho}=\bar{\rho}_{1}-\bar{\rho}_{2} x_{3 \min }$ the minimum value of the quantities $x_{3}^{1}, x_{3}^{2}$.

In the case of isotropic fluctuations of the refractive index is obtained a linear dependence on the distance along the middle ray.

$$
R_{\tau}\left(\bar{r}_{1}, \bar{r}_{2}\right)=\frac{x_{3 \min }}{2(\sqrt{\varepsilon})^{2}} \int_{0}^{\infty} R_{n}\left(\sqrt{\rho^{2}+\xi^{2}}\right) d \xi
$$

The correlation function $R_{\tau}$ of the eikonal is expressed through the spatial spectrum $\Phi_{n}(\varkappa)$ of fluctuations $n(\bar{r})$ according to the formula

$$
R_{\tau}\left(\bar{r}_{1}, \bar{r}_{2}\right)=\pi^{2} x_{3 \min } \int_{0}^{\infty} \Phi_{n}(\varkappa) J_{0}(\varkappa \rho) \varkappa d \varkappa(3.30)
$$

The dispersion of the eikonal, which is obtained from (3.30) at $\bar{\rho}=0, x_{3}^{1}, x_{3}^{2}=x_{3}$ increases linearly with increasing of distance along $x_{3}$

$$
\begin{aligned}
& D_{\tau}\left(x_{3}\right)=\sigma_{\tau}^{2}\left(x_{3}\right)=\frac{x_{3}}{2(\sqrt{\varepsilon})^{2}} \int_{0}^{\infty} R_{n}(\xi) d \xi= \\
& =\frac{\pi^{2} x_{3}}{2(\sqrt{\varepsilon})^{2}} \int_{0}^{\infty} \Phi_{n}(\varkappa) \varkappa d \varkappa
\end{aligned}
$$

Designating the effective integral correlations radius

$$
\begin{gathered}
\ell_{e f}=\int_{0}^{\infty} K_{n}(0, \xi) d \xi, \text { we represent (3.31) as } \\
\sigma_{\tau}^{2}\left(x_{3}\right)=\frac{x_{3}}{2(\sqrt{\varepsilon})^{2}} \int_{0}^{\infty} \sigma_{n}^{2} K_{n}(0, \xi) d \xi= \\
=\frac{x_{3}}{2(\sqrt{\varepsilon})^{2}} \sigma_{n}^{2} \ell_{e f}
\end{gathered}
$$

If $R_{n}(\rho)$ has a Gaussian shape

$$
R_{n}(\rho)=\sigma_{n}^{2} e^{-\rho^{2} / 2 \ell_{e f}^{2}}
$$

Then $\ell_{e f}=\sqrt{\pi / 2} \ell_{n}\left(\ell_{n}-\right.$ correlation radius of $n$ ), and the dispersion of the eikonal depends linearly on $x_{3}$ 


$$
\sigma_{\tau}^{2}\left(x_{3}\right)=\frac{\sqrt{\pi} \sigma_{n}^{2} \ell_{n}}{2 \sqrt{2}(\sqrt{\varepsilon})^{2}} x_{3}
$$

The spatial correlation can be represented as an expansion in the longitudinal and transverse correlation.

The longitudinal correlation $R_{I I}^{(\tau)}$ of the eikonal is calculated when the points $\overline{r_{1}}$ and $\overline{r_{2}}$ are located on one ray $\left(\bar{\rho}=\bar{\rho}_{1}-\bar{\rho}_{2}=0\right)$

$$
\begin{aligned}
& R_{I I}^{(\tau)}\left(x_{3}^{1}, x_{3}^{2}\right)=\frac{x_{3 \min }}{2(\sqrt{\varepsilon})^{2}} \int_{0}^{\infty} R_{n}(0, \xi) d \xi= \\
& =\frac{x_{3 \min }}{2(\sqrt{\varepsilon})^{2}} \sigma_{n}^{2} \ell_{e f}
\end{aligned}
$$

From (3.35) follows, that $R_{I I}^{(\tau)}$ doesn't depend on $x_{3 \max }$ from $\left(x_{3}^{1}\right.$ and $\left.x_{3}^{2}\right)$. Longitudinal correlation coefficient remains constant

$$
\begin{aligned}
& K_{I I}^{(\tau)}\left(x_{3}^{1}, x_{3}^{2}\right)=\frac{R_{I I}\left(x_{3}^{1}, x_{3}^{2}\right)}{\sigma_{\tau}\left(x_{3}^{1}\right) \sigma_{\tau}\left(x_{3}^{2}\right)}= \\
& =\sqrt{\frac{x_{3 \min }}{x_{3 \max }}}=\left\{\begin{array}{l}
\sqrt{x_{3}^{2} / x_{3}^{1}} a t x_{3}^{2}<x_{3}^{1} \\
\sqrt{x_{3}^{1} / x_{3}^{2}} \text { at } x_{3}^{2}>x_{3}^{1}
\end{array}\right.
\end{aligned}
$$

By fixing $x_{3}^{1}$ in the formula (3.36), we obtain, that the longitudinal correlation of the eikonal (phase) extends over a distance of about the path passed the ray $K_{I I} \sim x_{3}$. Fig. 3.2 shows the dependence $K_{I I}^{(\tau)}$ on $x_{3}^{2}$ at a fixed $x_{3}^{1}$

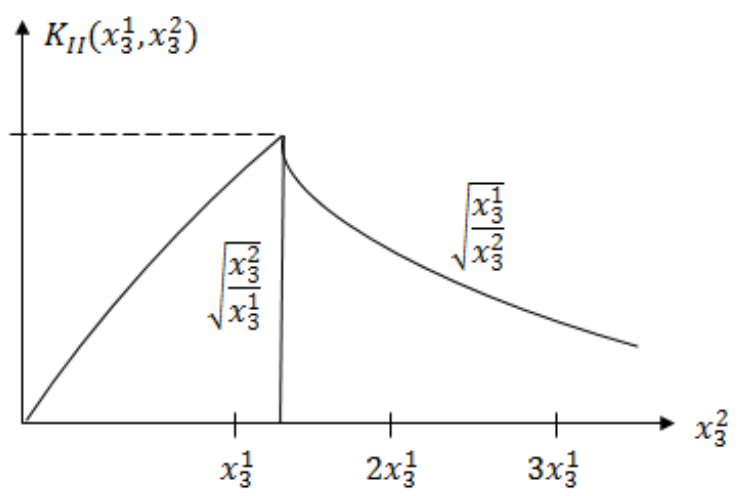

Figure 3.2. The dependence of longitudinal correlation coefficient on $x_{3}^{2}$
Cross-correlation $R_{\perp}(\bar{\rho}, L)$ at $x_{3}^{1}=x_{3}^{2}=L$ looks like

$$
R_{\perp}^{(\tau)}(\bar{\rho}, L)=\frac{L}{2(\sqrt{\varepsilon})^{2}} \int_{0}^{\infty} R_{n}(\bar{\rho}, \xi) d \xi
$$

In the case of isotropic Gaussian fluctuations

$$
R_{\perp}(\bar{\rho}, L)=\sigma_{\tau}^{2}(L) e^{-\rho^{2} / 2 \ell_{n}^{2}}
$$

As it follows from (3.38) the eikonal of rays bundle has a Gaussian correlation.

In the case of non-planar initial wave of Fig. 3.3

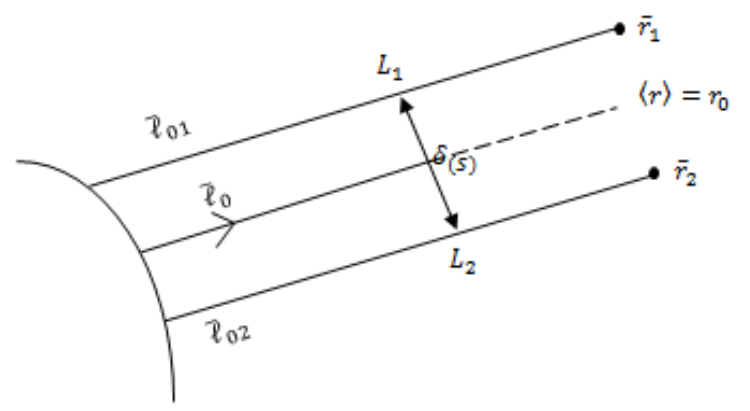

Figure 3.3. Case of non-planar initial wave

Where $L_{1}, L_{2}$ designate lengths of rays, coming to points $\bar{r}_{1}, \bar{r}_{2}$, across $\bar{\ell}_{01}, \bar{\ell}_{02}$ - unit vectors along these rays, $\delta(s)$ - the distance between the rays $\delta=|\bar{\rho}|=\left|\bar{\rho}_{1}-\bar{\rho}_{1}\right|$, then the correlation function of the eikonal is written in the form

$$
R_{\tau}\left(\bar{r}_{1}, \bar{r}_{2}\right)=\frac{L_{\min }}{2(\sqrt{\varepsilon})^{2}} \int_{0}^{\infty} R_{n}\left(\bar{\delta}+\ell_{0} \xi\right) d \xi
$$

The dispersion of the eikonal at $L_{1}=L_{2}=L, \delta(s)=0$ is calculated by the formula

$$
\sigma_{\tau}^{2}(L)=\frac{L}{2(\sqrt{\varepsilon})^{2}} \int_{0}^{\infty} R_{n}\left(\bar{\ell}_{0} \xi\right) d \xi
$$

As it follows from (3.39) correlation of the eikonal does not depend on the type of the wave shape and will be equal to the flat, spherical and cylindrical waves.

In a randomly inhomogeneous medium, which is statistically homogeneity and isotropic, the rays propagating in them are in average direct, orthogonal to the initial surface, and the wave surfaces (phase fronts) retain the semblance of an initial surface on average. We introduce on the unperturbed (average) surface the coordinate system $\alpha, \beta$ Fig. 3.4 


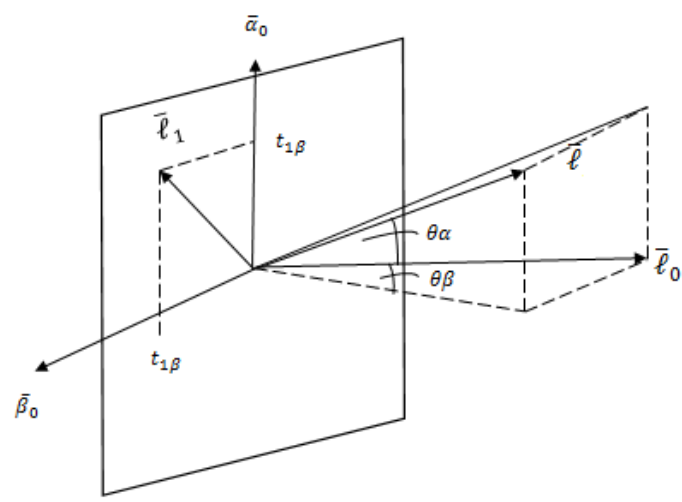

Figure 3.4. The unperturbed surface the coordinate system

With $\bar{\ell}_{0}$ we denote the unperturbed vector value tangential to the undisturbed ray and orthogonal to the unperturbed wave surface. Due to fluctuations in the front surface from the unperturbed surface normal to the front $\bar{\ell}$ will deviate from $\bar{\ell}_{0}$ by the value $\bar{\ell}_{1}$

$$
\bar{\ell}_{1}=\bar{\ell}-\bar{\ell}_{0}=\frac{\nabla_{\perp} \tau}{n}
$$

where $\nabla_{\perp}$ is the operator of cross-differentiation with respect to the undisturbed ray.

Vector $\bar{\ell}_{1} \perp \bar{\ell}_{0}$ lies in the plane tangent to the undisturbed wavefront $\tau_{0}=$ const .

We connect with the undisturbed ray Frenet trihedron formed by the unit vectors $\bar{\ell}_{0}, \bar{\alpha}_{0}, \bar{\beta}_{0}$ where $\bar{\ell}_{0}$ tangent, $\bar{\alpha}_{0}$ - of the principal normal, $\bar{\beta}_{0}$ - binormal vectors and submit

$$
\bar{\ell}_{1}=t_{1 \alpha} \bar{\alpha}_{0}+t_{1 \beta} \bar{\beta}_{0}
$$

Then up to the smallness of second order angles of arrival of the perturbed ray $\theta_{\alpha}, \theta_{\beta}$ will be equal

$$
\theta_{\alpha} \approx t_{1 \alpha}=\frac{1}{n}\left(\bar{\alpha}_{0} \nabla_{\perp} \tau_{1}\right)=\frac{1}{n} \frac{\partial \tau_{1}}{\partial \rho_{\alpha}}, \theta_{\beta}=\frac{1}{n} \frac{\partial \tau_{1}}{\partial \rho_{\beta}}
$$

From (3.43) follows, that the average values of the angles $\theta_{\alpha}, \theta_{\beta}$ taking into account $\tau_{1}=0$ will be equal to

$$
\theta_{\alpha}=\theta_{\beta}=0
$$

and correlation matrixes are given by expressions

$$
\begin{aligned}
& R_{\alpha \beta}^{\theta}\left(\bar{\rho}_{1}, \bar{\rho}_{2}\right)=\theta_{\alpha}\left(\bar{\rho}_{1}\right) \theta_{\beta}\left(\bar{\rho}_{2}\right)= \\
& =\frac{1}{n} \frac{\partial^{2} R_{\perp}\left(, \bar{\rho}_{2}\right)}{\partial \rho_{1 \alpha} \partial \rho_{2 \alpha}}
\end{aligned}
$$

Dispersions of angles $\theta_{\alpha}, \theta_{\beta}$ are equal to

$$
\theta_{\alpha s p h}^{2}=\frac{1}{3} \theta_{\alpha p l}^{2}
$$

From (3.46) follows, that the angles dispersion of a spherical wave arrival is less three times, than at a plane wave, that is caused by the fact that in the spherical wave rays propagate on average nearer to each other than in the plane.

A ray in a randomly nonuniform statistically homogeneous isotropic medium is a space curve, fluctuating around the middle (undisturbed) ray. The average value of fluctuations is determined by the mean square displacement of the ray from the unperturbed position. In the case of on average plane wave, coordinates of points of a ray trajectory are written as (3.1), then in the first approximation

$$
\overline{r_{1}}=\int_{0}^{L} \bar{\ell}_{1} d s=\int_{0}^{L} \nabla_{\perp} \tau_{1} d x_{3}^{1}
$$

From (3.47) follows, that a ray is displaced only in the transverse direction relative to the direction of the undisturbed ray. In (3.47) it is assumed that the initial surface is plane and the front is flat on average, propagating in the direction $x_{3}$, then $\overline{r_{1}}$, depends on $x_{1}, x_{2}$.

Calculating the elements of the correlation matrix we obtain

$$
\begin{aligned}
& R_{\alpha \beta}^{r}=(\rho, L)=R_{\perp}^{r}(\rho, L)\left(\delta_{\alpha \beta}-\frac{\rho_{\alpha} \rho_{\beta}}{\rho^{2}}\right)+ \\
& +R_{I I}^{r}(\rho, L) \frac{\rho_{\alpha} \rho_{\beta}}{\rho^{2}} \\
& R_{\perp}^{r}(\rho, L)=-\frac{L^{3}}{6} \int_{0}^{\infty} \frac{\partial^{2} R_{n}\left(\sqrt{\rho^{2}+\xi^{2}}\right)}{\partial \rho^{2}} d \xi \\
& R_{I I}^{r}(\rho, L)=-\frac{L^{3}}{6} \int_{0}^{\infty} \frac{\partial^{2} R_{n}\left(\sqrt{\rho^{2}+\xi^{2}}\right)}{\partial \rho} d \xi \\
& \rho=\sqrt{\rho_{\alpha}^{2}+\rho_{\beta}^{2}}
\end{aligned}
$$

From formulas (3.48), (3.49) follows that $r_{1 \alpha}\left(\bar{\rho}_{1}\right), r_{1 \beta}\left(\bar{\rho}_{2}\right)$ at $\bar{\rho}_{1}=\bar{\rho}_{2}$ are uncorrelated

$$
r_{1 \alpha} r_{1 \beta}=R_{\alpha \beta}^{r}(0)=0
$$

and mean squares (dispersions) are equal to each other, which is a consequence of fluctuations of the isotropic medium inhomogeneity 


$$
r_{1 \alpha p l}^{2}=r_{1 \beta}^{2}=R_{\alpha \alpha}^{r}(0)=-\frac{L^{2}}{6} \int_{0}^{\infty} \frac{R_{r}^{\prime}(\xi)}{\xi} d \xi
$$

In the case of a spherical wave

$$
r_{1 \alpha s p h}^{2}=\frac{1}{10} r_{1 \alpha p l}^{2}
$$

the mean square of the lateral displacement of the ray in a spherical wave is 10 times less than in a plane.

In a randomly inhomogeneous medium the signal propagation time (energy transfer) is determined by the group velocity

$$
C_{2 p}=C /\left(\frac{\partial(\omega n)}{\partial \omega}\right)^{-1}
$$

by group means

$$
\mathcal{L}=C \int_{0}^{L} \frac{d s}{C_{g r}}=\int_{0}^{L} \frac{\partial(\omega n)}{\partial \omega} d s
$$

In a statistically homogeneous isotropic medium $\varepsilon=$ const , which means that there is no regular refraction (bending) of the ray. Introducing $\mathcal{L}$ as

$$
\mathcal{L}=\sum_{K=0}^{\infty} \mathcal{L}_{k}
$$

in the first approximation we obtain

$$
\mathcal{L}_{1}=\frac{1}{2} \int_{0}^{L} \frac{\partial}{\partial \omega}\left(\omega \frac{\mathcal{V}}{\sqrt{\varepsilon}}\right) d s
$$

In the absence of a temporary (frequency) dispersion $\varepsilon$ and $\mathcal{V}$ are independent of $\omega$, then from (3.56) is obtained

$$
\mathcal{L}_{1}=\frac{1}{2} \int_{0}^{L}\left(\omega \frac{\mathcal{V}}{\sqrt{\varepsilon}}\right) d s=\tau_{1}
$$

From (3.57) follows that in this approximation the group path correction $\mathcal{L}_{1}$ coincides with the correction of the phase path (eikonal). This is a consequence of equality of the phase and group velocity in a statistically homogeneous isotropic medium. In particular, their dispersions are equal.

$$
\sigma_{\mathcal{L}}^{2}=\sigma_{\tau}^{2}=\frac{1}{2 \varepsilon} \int_{0}^{L} d s \int_{0}^{L} R_{n}\left[\bar{\ell}_{0} \xi ; \bar{r}_{0}(s)\right] d s
$$

Consider the second approach for the description of the ray propagation and of wave fronts in randomly inhomogeneous media. This approach is based on the theory of Markov' processes and allows us to describe the diffusion of rays.

We introduce on the initial surface the Cartesian coordinate system $x_{1}, x_{2}, x_{3}$ so that the axis $x_{3}$ has as direction vector the unperturbed ray, and $x_{1}, x_{2}$ are located in the tangent plane to $S_{t}^{0}$ normal to the ray $\bar{r}^{0}$.

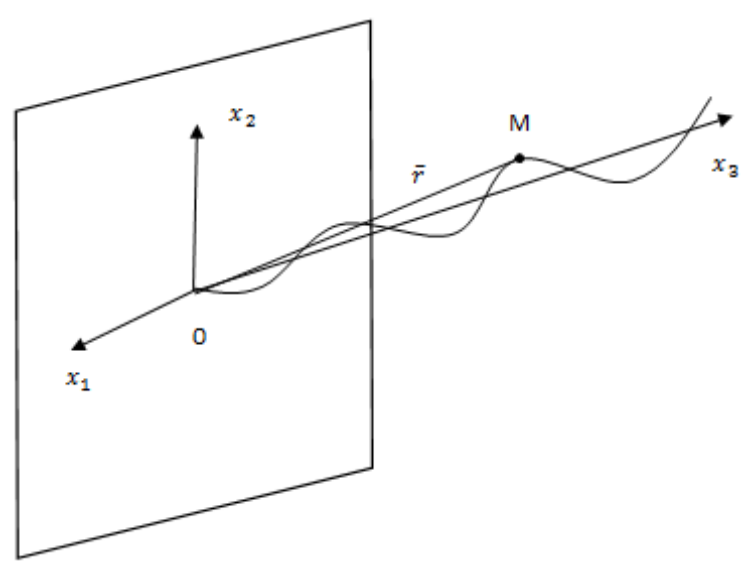

Figure 3.5. Scheme of ray fluctuation in inhomogeneous medium

The coordinate $\bar{r}$ of the arbitrary point $M$ of a curved ray can be represented as

$$
\bar{r}=\left(\bar{\rho}, x_{3}\right), \bar{\rho}=\left(x_{1}\left(x_{3}\right), x_{2}\left(x_{3}\right)\right)
$$

The equation describing the ray fluctuation can be written as

$$
\frac{d \bar{\rho}\left(x_{3}\right)}{d x_{3}}=\frac{\bar{\tau}_{\perp}\left(x_{3}\right)}{\sqrt{1-\bar{\tau}_{\perp}^{2}}}, \frac{d \bar{\tau}_{\perp}}{d x_{3}}=\frac{\bar{a}_{\perp}\left(\bar{\rho}, \bar{\tau}_{\perp}, x_{3}\right)}{\sqrt{1-\bar{\tau}_{\perp}^{2}}}
$$

where $\bar{\tau}_{\perp}=\left(\tau_{1}, \tau_{1}\right), \bar{a}_{\perp}\left(a_{1}, a_{2}\right), \bar{\tau}\left(\tau_{1}, \tau_{2}, \tau_{3}\right)$.

Equation (3.59) applies at $\bar{\tau}_{\perp}^{2}<1$. The case $\tau_{\perp}=1$ corresponds to the point the ray rotation on angle $\pi / 2$.

Considering that $\bar{\tau}_{\perp}^{2}<<1$, we will use the equation in the form of small-angle approximation

$$
\frac{d \bar{\rho}\left(x_{3}\right)}{d x_{3}}=\bar{\tau}_{\perp}\left(x_{3}\right), \frac{d \bar{\tau}_{\perp}\left(x_{3}\right)}{d x_{3}}=\nabla_{\perp} \mu\left(\bar{\rho}, x_{3}\right)
$$

where $\mu=\ln n$.

In an approximation of the diffusion random process the Einstein-Fokker equation (EFE) for the density probability has the form of [16-17]

$$
\frac{\partial P\left(\bar{\rho}, x_{3}\right)}{\partial x_{3}}+\bar{\tau}_{\perp} \frac{\partial P}{\partial \bar{\rho}}-D \frac{\partial^{2} P}{\partial \bar{\tau}_{\perp}^{2}}=0
$$

where $D$-diffusion coefficient

$$
D=\pi^{2} \int_{0}^{\infty} d \varkappa \varkappa^{2} \Phi(\varkappa)
$$

Here $\Phi(\varkappa)$ - three-dimensional spectral density of the correlation function for $\mu$. 
The initial condition for (3.61) has the form

$$
P_{0}\left(\bar{\rho}, \bar{\tau}_{\perp}\right)=\delta(\bar{\rho}) \delta\left(\bar{\tau}_{\perp}\right)
$$

The solution (3.61) with the initial condition (3.63) is a Gaussian distribution with the moments

$$
\begin{gathered}
\rho_{i}\left(x_{3}\right) \rho_{k}\left(x_{3}\right)=x_{i}\left(x_{3}\right) x_{k}\left(x_{3}\right)=\frac{2}{3} D \delta_{i k} x_{3}^{3}, i, k=1,2 \\
\rho_{i}\left(x_{3}\right) \tau_{\perp k}\left(x_{3}\right)=D \delta_{i k} x_{3}^{2}, \\
\tau_{\perp i}\left(x_{3}\right) \tau_{\perp k}\left(x_{3}\right)=2 D \delta_{i k} x_{3}
\end{gathered}
$$

The longitudinal correlation of the trajectories' points, located on one ray is calculated by the formula

$$
\bar{\rho}\left(x_{3}\right) \bar{\rho}\left(x_{3}^{1}\right)=2 D\left(x_{3}^{1}\right)^{2}\left(x_{3}-\frac{1}{3} x_{3}^{1}\right)
$$

and the correlation coefficient according to the formula

$$
\begin{aligned}
& \frac{\bar{\rho}\left(x_{3}\right) \rho\left(x_{3}^{1}\right)}{\sqrt{\bar{\rho}^{2}\left(x_{3}\right) \bar{\rho}^{2}\left(x_{3}^{1}\right)}}=\left(1+\frac{3}{2} \xi\right)(1+\xi)^{-3 / 2}, \\
& \xi=\frac{\left|x_{3}-x_{3}^{1}\right|}{x_{3 \min }}
\end{aligned}
$$

The joint diffusion of the two rays is described by a system of eight differential equations.

The system of equations for the coordinate points of ray trajectory has the form of

$$
\frac{d \bar{\rho}_{k}}{d x_{3}}=\bar{\tau}_{\perp k}, \frac{d \bar{\tau}_{\perp k}}{d x_{3}}=\frac{\partial \mu\left(\rho_{k}, x_{3}\right)}{\partial \bar{\rho}_{k}}
$$

where $k=1,2$ - ray numbers.

The density of probabilities, describing the relative diffusion of two rays

$$
\begin{aligned}
& P\left(\bar{\rho}^{*}, \bar{\ell}^{*}, x_{3}\right)= \\
& =\delta\left(\bar{\rho}_{1}-\bar{\rho}_{2}-\rho^{*}\right) \delta\left(\bar{\tau}_{\perp 1}-\bar{\tau}_{\perp 2}-\bar{\ell}^{*}\right)
\end{aligned}
$$

satisfies EFE, which has the form

$$
\begin{gathered}
\frac{\partial P}{\partial x_{3}}+\bar{\ell}^{*} \frac{\partial P}{\partial \bar{\rho}^{*}}-D_{\alpha \beta}\left(\bar{\rho}^{*}\right) \frac{\partial^{2} P}{\partial \ell_{\alpha}^{*} \partial \ell_{\beta}^{*}}=0 \\
D_{\alpha \beta}=2 \pi \int d \varkappa\left[1-\cos \bar{\varkappa} \bar{\rho}^{*}\right] \varkappa_{\alpha} \varkappa_{\beta} \Phi(\bar{\varkappa})
\end{gathered}
$$

In general, the equation (3.69) cannot be solved, however, for some models, you can obtain approximate solutions, from which we can draw certain conclusions concerning the spread of rays.

1. If $d_{n} \ll \rho$, then $D_{\alpha \beta}=2 D \delta_{\alpha \beta}$. This corresponds to the model, when the distance $\rho$ between rays on the initial surface is much larger than the correlation radius $d_{n}$ for a random field $\operatorname{grad} n$. Since in this case, their relative diffusion takes place with double diffusion coefficient, each ray diffuses independently of the other. The joint probability distribution in this case can be regarded as Gaussian.

2. If

$$
\begin{aligned}
& D_{\alpha \beta}(\bar{\rho})=\pi B\left(\rho^{2} \delta_{\alpha \beta}+2 \bar{\rho}_{\alpha} \bar{\rho}_{\beta}\right), \\
& B=\frac{\pi}{4} \int_{0}^{\infty} d \varkappa \varkappa^{3} \Phi(\varkappa)
\end{aligned}
$$

and EFE has the form of

$\frac{\partial P}{\partial x_{3}}+\bar{\ell}_{\alpha} \frac{\partial P}{\partial \bar{\rho}_{\alpha}}=\pi B\left(\rho^{2} \delta_{\alpha \beta}+2 \bar{\rho}_{\alpha} \bar{\rho}_{\beta}\right) \frac{\partial^{2} P}{\delta \ell_{\alpha} \partial \ell_{\beta}}=0(3.70)$

In this case, based on the EFE can obtain equations for $\bar{\rho}^{2}, \bar{\rho}, \bar{\ell}, \bar{\ell}^{2}$ the mean square distance between the rays in the area $A x_{3} \gg 1(A=16 \pi B)^{1 / 3}$ grows exponentially.

In the case of diffusion of $N$ rays, forming a ray tube is determined that the average cross-sectional area of the ray tube is retained in the plane $x^{3}=$ const, i.e. $S\left(x_{3}\right)=S_{0}$.

The end of the unit vector $\bar{\tau}$, tangent to the ray performs random walks along the unit sphere, depending on $t$ or $s$. We connect with the mobile point $M$ on the ray a fixed unit sphere so that the Cartesian coordinate axes $x_{1}, x_{2}, x_{3}$ with a center in point $M$ are moving parallel to a fixed coordinate system on the initial surface. The Frenet trihedron moving along the ray makes the rotational movements, which can be described in a spherical coordinate system Fig. 3.6



Figure 3.6. The Frenet trihedron in a spherical coordinate system 
Vector $\bar{\tau}$ has the components

$$
\begin{aligned}
& \bar{\tau}=\left(\tau_{1}, \tau_{2}, \tau_{3}\right)= \\
& =(\cos \varphi \sin \theta, \sin \varphi \sin \theta, \cos \theta)
\end{aligned}
$$

and equations for the ray can be written as

$$
\begin{gathered}
\frac{d x_{1}}{d s}=\cos \varphi \sin \theta, \frac{d x_{2}}{d s}=\sin \varphi \sin \theta, \frac{d x_{3}}{d s}=\cos \theta, \\
\frac{d \theta}{d s}=\cos \theta\left(\cos \varphi \frac{\partial \mu}{\partial x_{1}}+\sin \varphi \frac{\partial \mu}{\partial x_{2}}\right)-\sin \theta \frac{\partial \mu}{\partial x_{3}} \\
\frac{d \varphi}{d s}=-\frac{\sin \varphi}{\sin \theta} \frac{\partial \mu}{\partial x_{1}}+\frac{\cos \varphi}{\sin \theta} \frac{\partial \mu}{\partial x_{2}}
\end{gathered}
$$

Here $\mu=\ln n(\bar{r})$ is a random function.

The equations for the probability distribution functions $W(s, \bar{r}, \theta, \varphi)$ satisfy the Liouville equation, which describes the preservation of the volume in the phase space $[16,17]$. We introduce the probability density $P(s, \bar{r}, \theta, \varphi)$, connected with $W$ ratio $P(s, \bar{r}, \theta, \varphi)=W(\sin \theta)^{-1}$ then for $P(s, \bar{r}, \theta, \varphi)$ is performed the normalization condition

$$
\int_{V} d r \int_{0}^{\pi} \sin \theta d \theta \int_{-\pi}^{\pi} d \varphi P(s, \bar{r}, \theta, \varphi)=1
$$

and function $P(s, \bar{r}, \theta, \varphi)$ satisfies the equation

$$
\begin{aligned}
& \frac{\partial P}{\partial s}-\frac{\partial P}{\partial \theta}\left\{\frac{\partial \mu}{\partial x_{3}} \sin \theta-\left[\begin{array}{l}
\frac{\partial \mu}{\partial x_{1}} \cos \varphi+ \\
+\frac{\partial \mu}{\partial x_{2}} \sin \varphi
\end{array}\right] \cos \theta\right\}= \\
& =\bar{\tau}(2 P \nabla \mu-\nabla P)
\end{aligned}
$$

It is evident that the initial condition for (3.74) has the form

$$
\begin{aligned}
& P(s, \bar{r}, \theta, \varphi)=W_{0}\left(\bar{r}_{0}, \theta_{0}, \varphi_{0}\right)\left(\sin \theta_{0}\right)^{-1}= \\
& =\delta\left(r_{(0)}-r_{0}\right) \delta\left(\theta_{(0)}-\theta_{0}\right)\left(\sin \theta_{0}\right)^{-1}
\end{aligned}
$$

In the process of ray diffusion the probability density represents the average of the product of $\delta$ - functions random variables.

$$
\begin{aligned}
& P(s, \bar{r}, \theta, \varphi)=W(s, \bar{r}, \theta, \varphi)\left(\sin \theta_{0}\right)^{-1}= \\
& =\left(\sin \theta_{0}\right)^{-1} \delta(\bar{r}(s)-\bar{r}) \delta\left(\theta(s)-\theta_{0}\right) \delta(\varphi(s)-\varphi)
\end{aligned}
$$

In case, if the medium is statistically homogeneous isotropic, then the stationary ray mode of the fluctuation is possible, for which $P$ satisfies the equation

$$
\nabla P-2 P \nabla \mu=0
$$

Solution of (3.77) is written in the form

$$
P(s, \bar{r}, \theta, \varphi)=\frac{n^{2}(\bar{r})}{4 \pi \int d r n^{2}(\bar{r})}
$$

The average (3.78) in $n$, we obtain

$$
P(s, \bar{r}, \theta, \varphi)=\frac{n^{2}(\bar{r})}{4 \pi \int n^{2}(\bar{r}) d \bar{r}}
$$

From (3.79) follows that $P \sim n^{2}(\bar{r})$, i.e. the average density of the probability of arrival ray trajectories is longer than where $n^{2}(\bar{r})$ is higher, that means the bending of rays to the direction of increasing $n^{2}$. The same conclusion follows from the equation (3.77).

\section{Conclusions}

1. Stream lines of elastic energy in inhomogeneous media which have the tangent vector of the Poynting-Umov coincide with the rays which are the equations on basis of Fermat's principle.

2. Nonlinear differential equations for the trajectories of rays describe the occurrence of deterministic chaos of rays for certain types of inhomogeneous structures. In this case the rays behave randomly and it is therefore necessary to use the apparatus of stochastic functions and fields.

3. It was considered two models of inhomogeneous media which are equivalent to random inhomogeneous structures. The first model uses method of moments to determinate the coordinates of the trajectories of rays. In the second model, the equations for the probability are obtained when the medium properties are described by random functions of Markov type.

\section{A List of Symbols}

$\tau\left(M_{0}, M\right)$ - eiconal

$\bar{V}\left(x_{1}, x_{2}, x_{3}\right)$ - velocity

$\bar{\ell}$ - the unit vector directed along the ray

$\alpha, \beta$ - orthogonal curvilinear lines (rays and fronts)

$\theta(\alpha, \beta)$ - the angle between $\alpha$ and $\beta$ lines

$\bar{P}$ - vector of Poynting-Umov of the energy density

$E$ - the total energy density

$q_{i}$ - the generalized coordinates 
$p_{i}$ - the generalized impulses

$n\left(x_{1}, x_{2}, x_{3}\right)$ - the refractive medium index

$k$ - the curvature of the ray

$\chi$ - the torsion of the ray

$\theta$ - the angle between ray and grad $\mathrm{n}$

$\bar{n}$ - unit vector, the main normal

$\bar{b}$ - unit vector, the binormal

$\bar{\tau}$ - unit vector, the tangent

$\xi, \eta, s-$ the ray coordinates

$\Omega$ - the mean curvatures of the front

$K$ - Gaussian curvatures of the front

$I$ - the action

$\omega$ - the frequence

$R_{\tau}$ - correlation function

$\Phi_{n}(\chi)$ - spatial spectrum

$\ell_{n}$ - correlation radius

$\sigma_{\tau}^{2}-$ the dispersion

$p(s, \bar{r}, \theta, \varphi)$ - probability density

\section{REFERENCES}

[1] Friedhelm Kujpers. (1997) Klassische Mechanik. Wiley-VCH, Weinheim-Berlin-New

York-Chichester-Brisbane-Singapore-Toronto, Verlag $\mathrm{GmbH}$.

[2] Kravtsov Ju.A., Orlov Ju.I. (1991) Geometrical Optics of Inhomogeneous Media, in Springer Series of Wave Phenomena, V.6 Springer-Verlag.

[3] Haselgrove J. (1955) Ray Theory and New Method for Ray. Tracing. Proc. Camb. Conf. Phys. Ionosphere: 355-364.

[4] Chigarev A.V. (1980) A geometry of wave fronts in inhomogeneous media, Soviet Physics Acoustics. 26.6:
$905-912$.

[5] Rossikhin Ju.A., Shitikova M.V. (1994) A ray method of solving problems connected with a shock interaction, Acta Mechanica. 102: 103-121.

[6] Rossikhin Ju.A. (1986) Impact of a rigid sphere onto an elastic half-space. Sov. Appl. Mech., 22: 403-409.

[7] Rossikhin Ju.A., Shitikova M.V. (1995) Ray Methods for Solving Boundary Value Problems of Anisotropic Thermoelasticity with Thermal Relaxation, Appl. Mech. Rev. 48(1), 1-39.

[8] Whitham F.R.S. (1977) Linear and nonlinear waves, A Willey - interscience Publication, J. Willey \& Sons, London.

[9] Lighthill J. (1978) Waves in fluid, Cambridge University press, Cambridge-London-New York-Melbourne.

[10] Thomas T.Y. (1961) Plastic flow and fracture in solids. Academic Press New York

[11] Abdullaev S.S., Zaslavsky G.M. (1981) Nonlinear dynamics of rays in inhomogeneous media, J. Experimental and Theoretical Physics. 80.2: 525-536.

[12] Borisov A.V., Burenin A.A., Polenov V.S., Chigarev A.V. (2015) A wave dynamics of inhomogeneous and nonlinear media with application to geomechanics and biomechanics. Universum, Smolensk, 431.

[13] Chigarev A.V., Chigarev Ju.V. (1978) A possibility of a instability of rays in inhomogeneous media, Soviet Physics Acoustics. 24.6: 765-771.

[14] Iooss G., Joseph D.D. (1980) Elementary Stability and Bifurcation Theory, Springer-Verlag. New York-Heidelberg-Berlin.

[15] Babic V.M., Buldyrev V.S. (1991) Asymptotic Methods in Short-Wave Diffraction Theory, in Springer Series of Wave Phenomena, V. 4 Springer-Verlag, Berlin.

[16] Chigarev A.V. (2000) A stochastic and regular dynamics of inhomogeneous media. Technoprint, Minsk, 425.

[17] Van Kampen N.G. (1988) Stochastic Processes in Physics and Chemistry. North-Holland-Amsterdam-Oxford-New York-Tokyo. 